\title{
Chemical analysis of eight giant stars of the globular cluster NGC 6366
}

\author{
Arthur A. Puls,,${ }^{1 \star}$ Alan Alves-Brito, ${ }^{1 \star}$ Fabíola Campos,${ }^{2}$ Bruno Dias $^{3}$ \\ and Beatriz Barbuy ${ }^{4}$ \\ ${ }^{1}$ Departamento de Astronomia, Universidade Federal do Rio Grande do Sul, Av. Bento Gonalves 9500, Porto Alegre 91501-970, RS, Brazil \\ ${ }^{2}$ Department of Astronomy, University of Texas at Austin, Austin, TX 78712, USA \\ ${ }^{3}$ European Southern Observatory, Alonso de Cordova 3107, Santiago, Chile \\ ${ }^{4}$ Universidade de So Paulo, IAG, Rua do Mato 1226, Cidade Universitria, Sao Paulo 05508-900, Brazil
}

Accepted 2018 January 29. Received 2018 January 26; in original form 2017 August 28

\begin{abstract}
The metal-rich Galactic globular cluster NGC 6366 is the fifth closest to the Sun. Despite its interest, it has received scarce attention, and little is known about its internal structure. Its kinematics suggests a link to the halo, but its metallicity indicates otherwise. We present a detailed chemical analysis of eight giant stars of NGC 6366, using high-resolution and highquality spectra $(R>40000, \mathrm{~S} / \mathrm{N}>60)$ obtained at the VLT $(8.2 \mathrm{~m})$ and CFHT $(3.6 \mathrm{~m})$ telescopes. We attempted to characterize its chemistry and to search for evidence of multiple stellar populations. The atmospheric parameters were derived using the method of excitation and ionization equilibrium of $\mathrm{Fe}$ I and $\mathrm{Fe}_{\mathrm{II}}$ lines and from those atmospheric parameters we calculated the abundances for other elements and found that none of the elements measured presents star-to-star variation greater than the uncertainties. We compared the derived abundances with those of other globular clusters and field stars available in the literature. We determined a mean $[\mathrm{Fe} / \mathrm{H}]=-0.60 \pm 0.03$ for NGC 6366 and found some similarity of this object with $\mathrm{M} 71$, another inner halo globular cluster. The $\mathrm{Na}-\mathrm{O}$ anticorrelation extension is short and no star-to-star variation in $\mathrm{Al}$ is found. The presence of second generation stars is not evident in NGC 6366.
\end{abstract}

Key words: stars: abundances-Galaxy: abundances-globular clusters: individual: NGC 6366.

\section{INTRODUCTION}

Globular clusters (GCs) were, for many years, viewed as the prototypes of simple stellar populations, that is, all their stars would have the same age and metallicity in a first approximation. Nevertheless, it was known for decades that GCs-like 47 Tucanae have abundant variations in some light elements, which could be explained by primordial origin or some unknown mixing mechanism (e.g. Hesser \& Bell 1980). A paradigm shift began to occur when the $\mathrm{Na}-\mathrm{O}$ and $\mathrm{Mg}-\mathrm{Al}$ anticorrelations, already known for Red $\mathrm{Gi}-$ ant Branch (RGB) stars (e.g. Gratton, Sneden \& Carretta 2004; Gratton, Carretta \& Bragaglia 2012), were also found in Sub-Giant Branch (SGB) and Main-Sequence (MS) stars that did not suffer any mixing process (e.g. Gratton et al. 2001). Since stellar evolution models do not account for these observational results, the hypothesis of multiple stellar populations gained strength. Afterwards, multiple populations began to be identified in high-precision photometric observations, first in $\omega$ Centauri (Bedin et al. 2004), which is the brightest Galactic GC, and new photometric techniques are being

^E-mail: puls.arthur@gmail.com (AAP); alan.brito@ufrgs.br (AAB) developed to study GCs multiple populations (see e.g. Milone et al. 2017). As of today, the consensus is that some of the light elements ( $\mathrm{Li}, \mathrm{CNO}, \mathrm{Na}, \mathrm{Al}, \mathrm{Mg}$, and $\mathrm{F}$ ) studied in GCs present star-to-star abundance variations. There is no consensus yet about the nature of the polluters, with the main candidates being intermediate-mass Asymptotic Giant Branch (AGB) stars (Ventura et al. 2001), fastrotating massive stars (Decressin et al. 2007), massive interacting binaries (de Mink et al. 2009), and supermassive stars (Denissenkov $\&$ Hartwick 2014). However, Renzini et al. (2015) argue that three of these four models violate the constraints imposed by the observations, and only the AGB scenario could explain the multiple populations, but only if its flaws - like the difficulty in reproducing the observed $\mathrm{Na}-\mathrm{O}$ anticorrelation - can be fixed.

Despite being important for calibration of metallicity and abundance ratio studies based on low-resolution spectra and/or population synthesis of star clusters, few metal-rich Galactic GCs have been studied with high-resolution and high $\mathrm{S} / \mathrm{N}$ ratio spectra to date. In this sense, NGC 6366 (Galactic coordinates $l=18.4085 \mathrm{deg}, b$ $=+16.0356 \mathrm{deg}$ ) is an interesting object to be investigated. It is the fifth closest GC according to the catalogue of Harris (1996, 2010 edition), with $R_{\odot}=3.5 \mathrm{kpc}$. Using isochrone fitting, Campos et al. (2013) found $(m-M)_{V}=15.02$ with $A_{V} \approx 2.11$. Also, this GC still 
Table 1. Program stars, coordinates, distance to the geometric centre of the GC and photometry used (see the text for references).

\begin{tabular}{|c|c|c|c|c|c|c|c|c|}
\hline $\begin{array}{l}\text { ID } \\
\text { (this work) }\end{array}$ & $\begin{array}{c}\text { ID } \\
\text { (Dias et al. 2016) }\end{array}$ & $\begin{array}{c}\text { RA } \\
\text { (hh:mm:ss) }\end{array}$ & $\begin{array}{c}\text { Dec } \\
\text { (dd:mm:ss) }\end{array}$ & $\begin{array}{c}\mathrm{r}_{\mathrm{pos}} \\
(\operatorname{arcmin})\end{array}$ & $\begin{array}{c}V \\
(\mathrm{mag})\end{array}$ & $\begin{array}{c}J \\
(\mathrm{mag})\end{array}$ & $\begin{array}{c}H \\
(\mathrm{mag})\end{array}$ & $\begin{array}{c}K_{s} \\
(\mathrm{mag})\end{array}$ \\
\hline \multicolumn{9}{|c|}{ UVES@VLT } \\
\hline 01 & $\ldots$ & 172758.32 & -050429.35 & 3.53 & 15.731 & 12.517 & 11.837 & 11.680 \\
\hline 03 & $\ldots$ & 172757.79 & -050815.40 & 4.85 & 15.607 & 12.107 & 11.316 & 11.078 \\
\hline 05 & $\ldots$ & 172754.84 & -050154.80 & 3.91 & 15.807 & 12.472 & 11.760 & 11.597 \\
\hline 06 & $\ldots$ & 172740.93 & -050201.93 & 2.88 & 15.650 & 12.398 & 11.677 & 11.495 \\
\hline 07 & $\ldots$ & 172734.30 & -050109.66 & 4.40 & 15.858 & 12.468 & 11.777 & 11.555 \\
\hline 08 & $\ldots$ & 172727.03 & -050316.70 & 4.56 & 15.713 & 12.464 & 11.812 & 11.622 \\
\hline \multicolumn{9}{|c|}{ ESPaDOnS@CFHT } \\
\hline 11 & 15 & 172743.69 & -050630.30 & 1.72 & 14.305 & 10.637 & 9.787 & 9.589 \\
\hline 18 & 05 & 172744.48 & -050238.10 & 2.16 & 14.697 & 10.985 & 10.157 & 9.946 \\
\hline
\end{tabular}

has no measurements based on high-resolution spectra of chemical elements widely studied in the context of Galactic and GC chemical evolution, such as $\mathrm{Al}$ and Eu.

Previous photometric and spectroscopic studies have found a range of $[\mathrm{Fe} / \mathrm{H}]$ values for NGC 6366. The first determination was from Pike (1976), $[\mathrm{M} / \mathrm{H}]=-0.50 \pm 0.20$, using the Hartwick (1968) S parameter, derived from the colour-magnitude diagram (CMD) morphology. Later, Da Costa \& Armandroff (1995) found $[\mathrm{Fe} / \mathrm{H}]=-0.65 \pm 0.07$ using fits in the Ca II triplet in the nearinfrared, while a more recent analysis based on low-resolution data from Dias et al. (2016) measured $[\mathrm{Fe} / \mathrm{H}]=-0.61 \pm 0.05$, through spectrum fitting from two different spectral libraries. Johnson et al. (2016) published the first high-resolution $(R \approx 38000)$ work on NGC 6366. From atmospheric model measurements of red giants, they estimated $[\mathrm{Fe} / \mathrm{H}]=-0.55$ for this cluster. The mean value given in the Harris catalogue is $[\mathrm{Fe} / \mathrm{H}]=-0.59$.

Being metal-rich and near the outer bulge, NGC 6366 may be chemically associated with this Galactic component. Usually, bulge GCs have $[\mathrm{Fe} / \mathrm{H}]>-1.3$ (Bica, Ortolani \& Barbuy 2016). Also, they pass frequently in the high-density regions of the Galaxy, being subject to tidal stripping, which seems to be the case of NGC 6366 (Paust et al. 2009). According to Da Costa \& Seitzer (1989), NGC 6366 has halo kinematics, and is 'unambiguously' associated with that Galactic component, so it could be either a metal-rich GC from the inner halo or a bulge GC which suffered kinematic heating while evolving with the Galaxy.

Campos et al. (2013) calculated an age of $11 \pm 1.15$ Gyr for this GC, pointing out that the presence of multiple populations or $\mathrm{CNO}$ enhancement could affect the age estimation. However, the presence or absence of multiple stellar populations in NGC 6366 is currently an open question. Based on a photometric index, $(U$ $B)-(B-I)$, which is sensitive to the abundances of helium and light elements, Monelli et al. (2013) suggest a bimodality in the RGB of NGC 6366.

The goal of this paper is to present a detailed chemical abundance analysis of bright giant stars in NGC 6366 based on the highest resolution spectra obtained to date for this $\mathrm{GC}$. We have derived chemical abundances for 16 species (O, Na, Al, Si, Ca, Sc, Ti, V, Cr, $\mathrm{Mn}, \mathrm{Fe}, \mathrm{Co}, \mathrm{Ni}, \mathrm{Y}, \mathrm{Ba}$, and $\mathrm{Eu}$ ), including the first measurement of $\mathrm{Al}$, that is crucial for the analysis of multiple populations. Our work provides additional observational constraints to the understanding of the chemical evolution of the Galaxy and of the GC chemical evolution itself.

In Section 2 we describe the observations and the data-reduction process. Section 3 presents the method of determination of radial

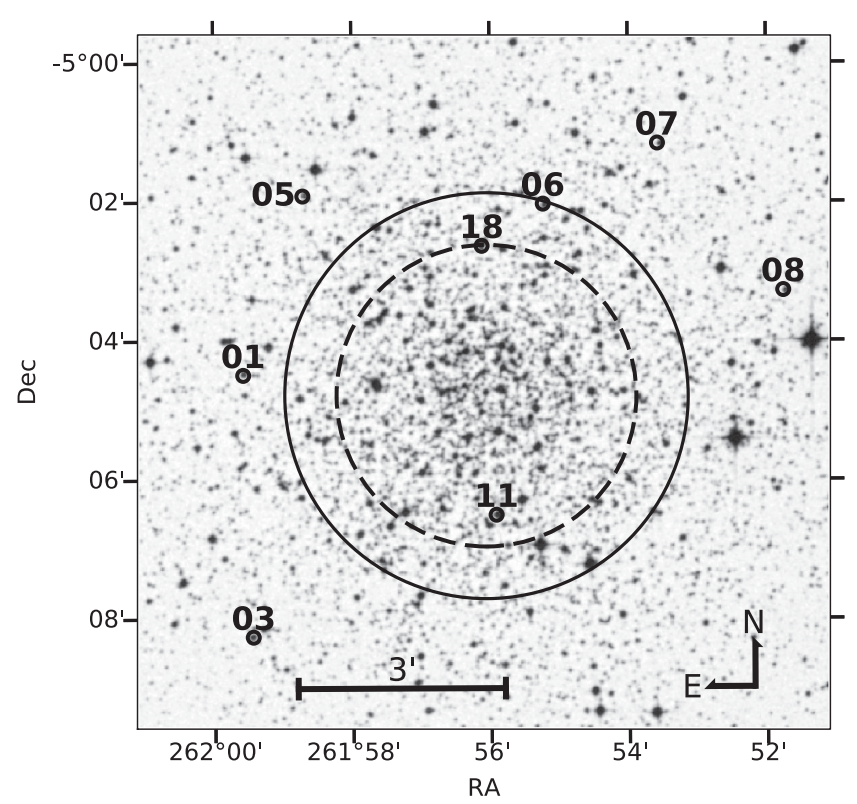

Figure 1. Finding chart for NGC 6366 with the sample stars labeled. The dashed and solid circumferences delimit, respectively, the core radius and the half-light radius, taken from Harris (1996, 2010 edition). Image from the Digitized Sky Survey.

velocities, atmospheric parameters, and chemical abundances. Results and discussion of the data set are presented in Section 4 and, in Section 5, we make final remarks.

\section{OBSERVATIONS AND DATA REDUCTION}

Our sample consists of eight bright giant stars (see Table 1 and Fig. 1 for details). The two brightest targets - stars 11 and $18-$ were selected from Pike (1976), where their IDs are III-11 and I-18, respectively. Their spectra were acquired with ESPaDOnS, which is a high-dispersion echelle spectrograph and spectropolarimeter mounted at the 3.6-m Canada France Hawaii Telescope (CFHT). The observations were carried out on two nights of 2015 March and July, in the star+sky mode. The exposure times were $3 \times 2315 \mathrm{~s}$ per star, reaching $R \sim 68000$. The observed wavelength ranges from ultraviolet (3699 $\AA$ ) to near-infra-red wavelengths (10483 $\AA$ ). ESPaDOnS data are reduced through the software Upena/Libre-Esprit pipeline, provided by the instrument team (Donati et al. 1997). Both 
stars 11 and 18 are also included in the analysis of Dias et al. (2016), where their IDs are 15 and 05, respectively.

The remaining targets were acquired from the ESO archive. They were observed with the Ultraviolet and Visual Echelle Spectrograph (UVES) (Dekker et al. 2000) at the Very Large Telescope (VLT, four 8.2-m telescopes) for a study of metal-rich Galactic GCs (Feltzing, Primas \& Johnson 2009). An 1 arcsec slit was used, yielding a resolution $R \sim 40000$. The exposure times are $2 \times 3300 \mathrm{~s}$, except in the blue region (4727-5804 $\AA$ ) of stars 05 and 06 , which have a single exposure each. For stars 03 and 07 only the red portion of the spectra, which covers wavelengths from 5818 to $6835 \AA$, is available. All spectra were taken directly from the archive. We only needed to normalize the UVES spectra and to correct all of them for Doppler shift. These tasks were made with standard IRAF routines. After proceeding with the Doppler shift correction, spectra with multiple exposures were co-added to increase the signal-tonoise ratio $(\mathrm{S} / \mathrm{N})$ of individual stars in both subsamples. Typical values of $\mathrm{S} / \mathrm{N}$ are 80 per pixel for the UVES@VLT subsample and 60 per pixel for the ESPaDOnS@CFHT stars, measured at $\lambda \approx 6150 \AA$. We estimate $\mathrm{S} / \mathrm{N} \sim 25$ at $\lambda \approx 5000 \AA$ in ESPaDOnS spectra - see Section 3 for a discussion about the implications of lower S/N. Fig. 2 shows a portion of all spectra.

The spatial distribution of the sample is shown in Fig. 1, while in Fig. 3 we show the location of the sample in a $K_{\mathrm{s}}$ versus $\left(J-K_{\mathrm{s}}\right)$ CMD. The photometry of $J, H$, and $K_{\mathrm{s}}$ bands is from the Two Micron All Sky Survey (2MASS) catalogue (Skrutskie et al. 2006). In the $V$ band, the photometric data for star 06 is from the Naval Observatory Merged Astrometric Data set (NOMAD/YB6) catalogue (Zacharias et al. 2004). For stars 01 and 11, the $V$ photometry is from Stetson (2000). Otherwise, the photometric data in the $V$ band are from Sariya \& Yadav (2015).

\section{ANALYSIS}

The heliocentric radial velocities were determined using the IRAF rvidlines task, by measuring the positions of a large number of atomic absorption lines in each spectrum. In the UVES subsample, the adopted values are from the red part of each spectrum - because two stars lack the blue part, as pointed out previously - with 69 absorption features measured. In the ESPaDOnS subsample, 217 lines were measured along all spectra. For each object, the final value of radial velocity is the mean of the values obtained for each exposure. The uncertainties of the radial velocities of each star were propagated from the standard deviation of the mean of the measurements from each exposure.

The stellar atmospheric parameters - effective temperature $\left(T_{\text {eff }}\right)$, surface gravity $(\log g)$, metallicity $([\mathrm{Fe} / \mathrm{H}])$, and microturbulent velocity $\left(\xi_{\mathrm{t}}\right)$ - which are essential in a chemical abundance analysis, were obtained by photometric (for $T_{\text {eff }}$ and $\log g$ ) and spectroscopic methods (for all). The photometric $T_{\text {eff }}$ were calculated using dereddened $\left(J-K_{\mathrm{s}}\right)$ and $\left(V-K_{\mathrm{s}}\right)$ colours employing the calibrations of Alonso, Arribas \& Martínez-Roger (1999, see Alonso, Arribas \& Martínez-Roger 2001 for an erratum). The colour-temperature $\left(V-K_{\mathrm{s}}\right)$ calibration was chosen for first guesses because it has the lowest mean variation among all calibrations - an error of 0.05 in magnitude implies an error of 0.7 per cent in $T_{\text {eff }}$. As the colourtemperature $(J-K)$ calibration is independent of $[\mathrm{Fe} / \mathrm{H}]$, its effective temperatures and surface gravity values were used as a control group. The 2MASS colours were transformed to the CIT system (Carpenter 2001) and then to the TCS system (Alonso, Arribas \& Martinez-Roger 1998). We adopted $E(B-V)=0.71$ and, initially, $[\mathrm{Fe} / \mathrm{H}]=-0.59$ (Harris 1996, 2010 edition). The relations

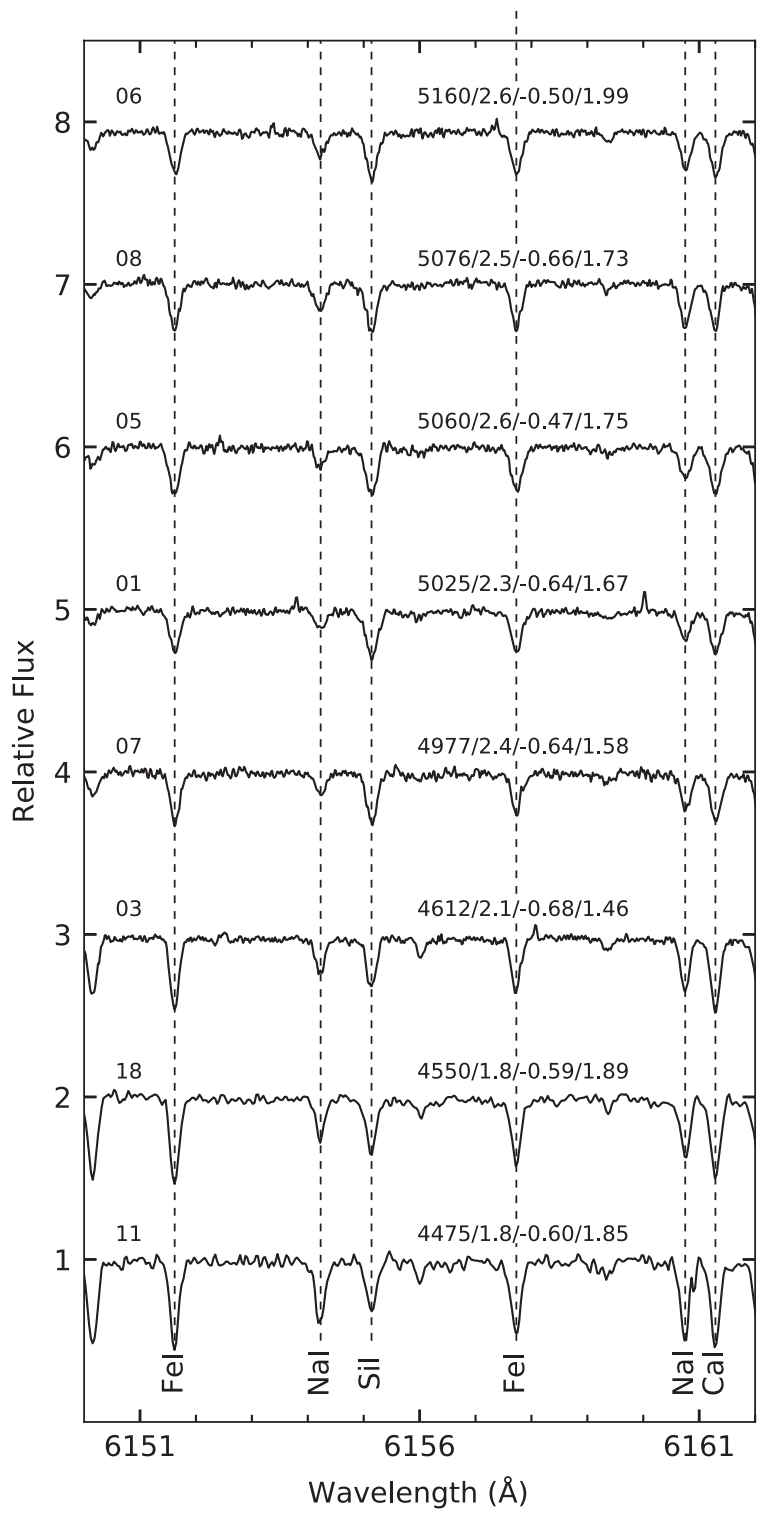

Figure 2. A portion of the spectra with some of the lines measured in this work identified. Above each spectrum are the IDs of the stars and the respective spectroscopic atmospheric parameters $\mathrm{T}_{\text {eff }}(\mathrm{K}), \log g(\mathrm{dex})$, $[\mathrm{Fe} / \mathrm{H}](\mathrm{dex})$, and $\xi_{\mathrm{t}}\left(\mathrm{km} \mathrm{s}^{-1}\right)$. The spectra are ordered by decreasing $T_{\text {eff }}$.

$\mathrm{E}(V-K) / E(B-V)=2.744$ and $\mathrm{E}(J-K) / E(B-V)=0.525$ (Rieke $\&$ Lebofsky 1985) were used for extinction estimation. Campos et al. (2013) built a detailed reddening map of NGC 6366, but the map comprises the central region of the GC only, thus we have no detailed information about differential reddening in the outer regions where our sample stars are located, and a constant value of $E(B-V)$ was chosen.

To estimate photometric $\log g$, we used the relation:

$$
\begin{aligned}
\log \left(g_{*}\right)= & \log \left(\frac{\mu_{*}}{\mu_{\odot}}\right)+4 \log \left(\frac{T_{\text {eff } *}}{T_{\text {eff } \odot}}\right) \\
& +\frac{2}{5}\left(M_{\mathrm{bol}, *}-M_{\mathrm{bol}, \odot}\right)+\log \left(g_{\odot}\right),
\end{aligned}
$$

where $\mu_{*}$ is stellar mass and $M_{\mathrm{bol}}$ the bolometric magnitude. To perform the calculations, we assumed mass of $0.8 \mathrm{M}_{\odot}$ for all stars from inspection of an 11-Gyr PARSEC isochrone (Bressan et al. 


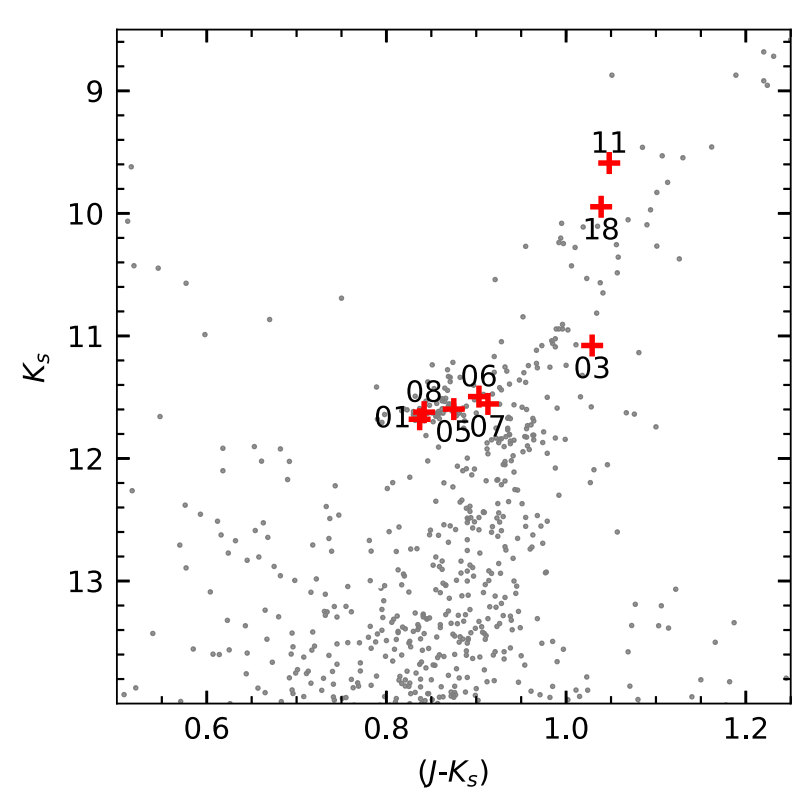

Figure 3. A $K s$ versus $\left(J-K_{\mathrm{S}}\right)$ CMD of NGC 6366. Red crosses: stars studied in this work. Grey dots: 2MASS point sources within $10 \mathrm{arcmin}$ from the centre of the GC.

2012) with global metallicity $Z=0.0039^{1}$ and RGB mass-loss coefficient $\eta_{\text {Reimers }}=0.2$. The surface gravities of the Horizontal Branch (HB) stars change by 0.06 dex if we change $\eta_{\text {Reimers }}$ from 0.2 to 0.4 . The bolometric magnitude was estimated with the method described in Torres (2010, equation (7)). The values adopted for

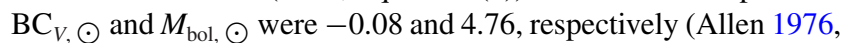
with $M_{\mathrm{bol}} \odot$ corrected as recommended by Torres).

After the derivation of the $[\mathrm{Fe} / \mathrm{H}]$ for each star using the spectroscopic method, described hereafter, we recalculated the $(V-K)$ effective temperatures and surface gravities inserting the $[\mathrm{Fe} / \mathrm{H}]$ values derived spectroscopically into the colour- $T_{\text {eff }}$ calibration, star by star. As the $(J-K)$ calibration is metallicity independent, it was not necessary to recalculate its photometric parameters.

The spectroscopic stellar parameters for each object were determined by traditional methods. Equivalent widths (EWs) of $\mathrm{Fe}_{\mathrm{I}}$ and $\mathrm{Fe}$ II lines were measured by hand using IRAF splot task. Those features with EW > $160 \mathrm{~m} \AA$ were discarded, since stronger lines do not possess the Gaussian profiles needed for our analysis. For weaker lines, lower $\mathrm{S} / \mathrm{N}$ makes the measurement difficult. If we define the $\mathrm{S} / \mathrm{N}$ of a normalized spectrum in a given region as the inverse of the standard deviation $(1 \sigma)$ of the continuum noise, and assume that the minimum depth for a line to be distinguishable from continuum noise is $3 \sigma$, the minimum $\mathrm{EW}$ for a Gaussian absorption feature be considered in our analysis is $\sim 19 \mathrm{~m} \AA$ at $\mathrm{S} / \mathrm{N}=25(\lambda \approx 5000 \AA$ in ESPaDOnS spectra, as discussed in Section 2) for a typical FWHM of $150 \mathrm{~m} \AA$. No lines with $\mathrm{EW}<19 \mathrm{~m} \AA$ were considered for the stars observed with ESPaDOnS, and no lines with EW $<40 \mathrm{~m} \AA$ were taken into account in the bluest $(\lambda<5000 \AA)$ regions of their spectra.

For each star, an LTE 1D plane-parallel atmospheric model was built with the ATLAS9 code (Castelli, Gratton \& Kurucz 1997), assuming a solar $\alpha$ mixture, and used as input in the abfind driver of MOOG (Sneden 1973). The expected difference in abundances be-

${ }^{1} Z_{\odot}=0.0152$
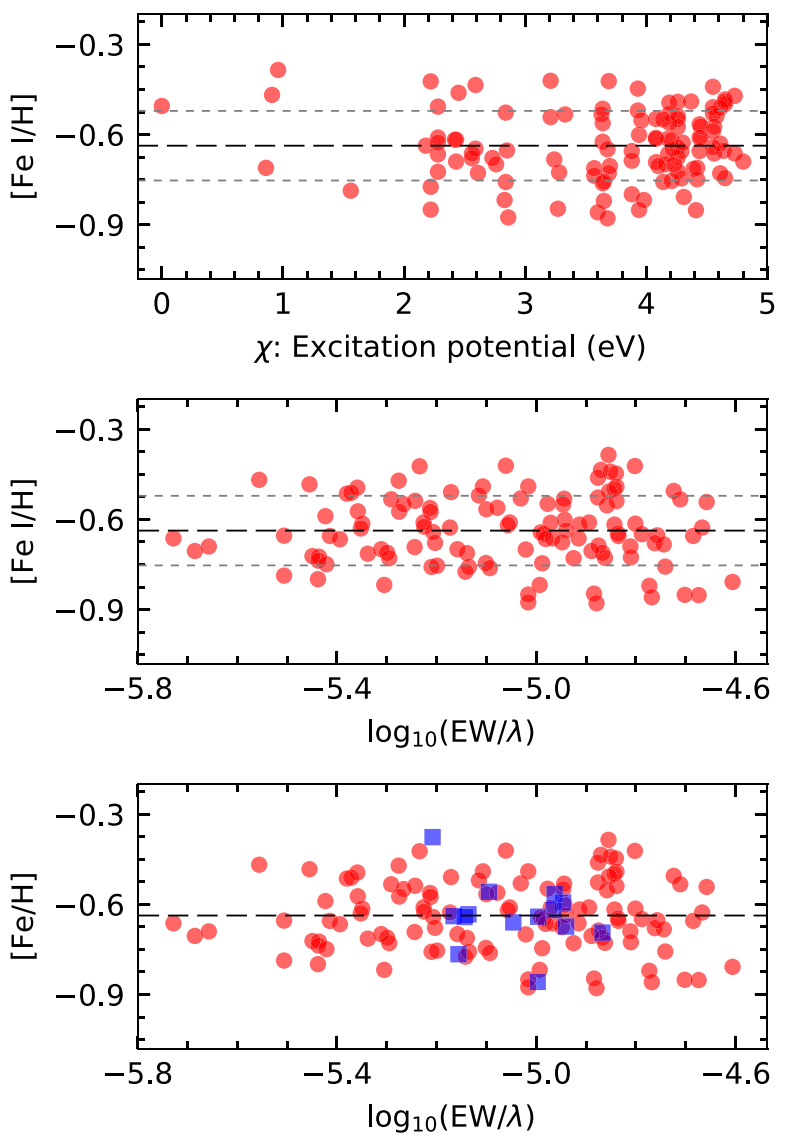

Figure 4. Diagrams showing the end of the iterative process used to find the spectroscopic atmospheric parameters for star 01. Top: Excitation equilibrium ( $\chi$ versus abundance) diagram, employed to find the spectroscopic effective temperature when the slope of the least-squares fit is zero. Centre: Reduced width $(\mathrm{EW} / \lambda)$ versus abundance diagram. Used to find the microturbulence velocity also when the slope of the least-squares fit is zero. Bottom: Ionization equilibrium ( $\mathrm{Fe}_{\mathrm{I}}$ versus $\mathrm{Fe}$ II) diagram. Employed to find the spectroscopic surface gravity when abundances of both $\mathrm{Fe}_{\mathrm{I}}$ and $\mathrm{Fe}$ II species are equal, where red circles and blue squares represent $\mathrm{Fe}$ I and $\mathrm{Fe}$ II absorption features, respectively. Black dashed lines are the least-squares fit - when their slope is zero their $y$-value equals Fe I abundance. Grey dotted lines are $1 \sigma$ limits. In the bottom plot, the black dotted line indicates the least squares fit to both $\mathrm{Fe}$ I and $\mathrm{Fe}$ II points.

tween plane-parallel and spherical models is negligible $(\sim 0.02$ dex; see e.g. Alves-Brito et al. (2010)). The effective temperature $\left(T_{\text {eff }}\right)$ was found by removing any trends in plots of $\mathrm{Fe}$ I abundance versus line excitation potential $(\chi)$ - excitation equilibrium. The same approach was used in plots of Fe I abundance versus reduced EW (log $(\mathrm{EW} / \lambda))$ to find the microturbulent velocity $\xi_{\mathrm{t}}$. By forcing the abundances of $\mathrm{Fe} \mathrm{I}$ and $\mathrm{Fe}$ II to be equal, we obtained the surface gravity in terms of the ionization equilibrium. Finally, the metallicity $[\mathrm{Fe} / \mathrm{H}]$ was determined by equalizing the value used in the model and the value given by MOOG in the output. The adopted stellar parameters were found when all the conditions were satisfied simultaneously, after an iterative process. Examples of excitation equilibrium diagrams are given in Fig. 4. The Fe $\mathrm{I}$ abundances are subject to NLTE effects which could affect the values of the atmospheric parameters, particularly the surface gravity. However, the use of the LTE approximation is justified as these effects are negligible for cold giants in the considered metallicity regime $([\mathrm{Fe} / \mathrm{H}] \sim-0.60)$, and NLTE corrections would be small if compared to the adopted un- 


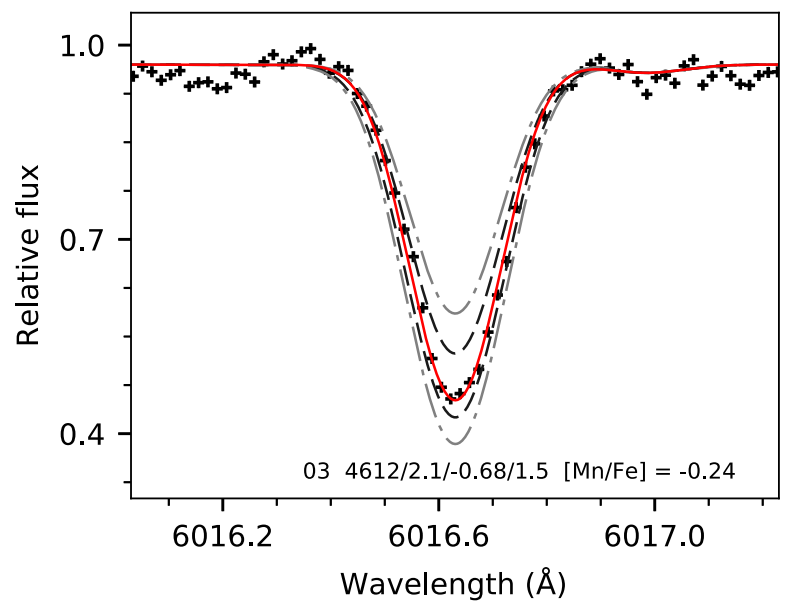

Figure 5. Example of spectral synthesis of the $6016 \AA$ line of Mn. The points are the observed spectra. The solid red line is the best-fitting, while the grey lines represent the upper and lower uncertainties in $[\mathrm{Mn} / \mathrm{Fe}]$ (dashed dark grey for $1 \sigma$, dash-dot light grey for $2 \sigma$ ). Star ID, the four atmospheric parameters, and the mean abundance of all lines measured for the star are labelled in the figure.

certainties (see figs 2, 4, 6, and 7 of Lind, Bergemann \& Asplund 2012 , and references therein).

To obtain the abundance of elements with atomic number $Z \leq$ 28 , we adopted EW measurements used as input in the abfind driver of MOOG, except for Mn. For heavier elements and Mn, the spectral synthesis method was used by employing the synth driver of MOoG. The spectral synthesis method consists of comparing absorption lines of synthetic and observed spectra, where the former are built from the chosen atmospheric model for each star. The chemical abundance of a given element $\mathrm{X}$ is found varying $[\mathrm{X} / \mathrm{Fe}]$ in the input to make the synthetic and observed line profiles match. Fig. 5 shows a visualization of the method (see also Figs A1, A2, A3).

The adopted line list for all elements is the same as given in Yong et al. (2014), except for Mn, where we adopted the hyperfine structure derived by Barbuy et al. (2013) for the Mn $6000 \AA$ A triplet. For Eu we used the same hyperfine structure of Marino et al. (2011, sent by private communication). Table 2 presents the atomic data of $\log g f$ and $\chi$ and EW measurements for individual lines of the elements up to Ni. The chemical abundances of the lines measured by spectral synthesis are in Table 3 .

\subsection{Uncertainties}

For each star, after converging the values of the atmospheric parameters to the adopted figures, the abundances of Fe show a dispersion around the mean (see Fig. 4). To estimate the uncertainties of each atmospheric parameter, we varied one atmospheric parameter at a time until the abundance of $\mathrm{Fe} I$ returned in the MOOG output changed by a standard deviation. The atmospheric parameters uncertainties adopted in this work are typical values found in these estimates. The values are $\Delta T_{\text {eff }} \approx \pm 150 \mathrm{~K}, \Delta \log g \approx \pm 0.3 \mathrm{dex}, \Delta[\mathrm{Fe} / \mathrm{H}] \approx$ $\pm 0.1 \mathrm{dex}$, and $\Delta \xi_{\mathrm{t}} \approx \pm 0.2 \mathrm{~km} \mathrm{~s}^{-1}$. To estimate the uncertainties in chemical abundances, we created atmospheric models varying only one parameter by its uncertainty value per new model created. The sensitivities of the abundance values due to each atmospheric parameter variation were treated as the uncertainties for each element related to that atmospheric parameter variation and were propagated by quadrature. Then, the value was propagated again by quadrature with the standard deviation of line-by-line abundances given by MOOG to get the total uncertainties for elements measured from EW (see Table A1).

The sensitivities of the abundances to variations of the stellar atmospheric parameters of two representative stars - one from the $\mathrm{HB}$ and another one from the cold giants - are shown in Table 4. The uncertainties for elements measured by spectral synthesis are from these two representative stars: the uncertainties for Mn, Y, Ba, and Eu of the UVES subsample were taken from the sensitivities of star 01, while the uncertainties for these elements of the ESPaDOnS subsample were taken from sensitivities of star 18. We also defined the ratio $\rho=\sigma_{\mathrm{cl}} /\left\langle\sigma_{*}\right\rangle$, where $\sigma_{\mathrm{cl}}$ and $\left\langle\sigma_{*}\right\rangle$ are, respectively, the standard deviation of the star-to-star variation of a certain element in the GC and the mean uncertainty of this element.

\section{RESULTS AND DISCUSSION}

In this section we present and discuss our results on kinematics and chemical abundances. The $\alpha$ elements, Fe-peak, light odd-Z elements, and those synthesized by neutron capture are discussed individually. The final abundance pattern will be compared with those displayed by stars in different environments, field, and other GCs.

\subsection{Kinematics}

The mean heliocentric radial velocity (RV) for our sample is $-121.2 \pm 0.7 \mathrm{~km} \mathrm{~s}^{-1}\left(\sigma=2.0 \mathrm{~km} \mathrm{~s}^{-1}\right)$. Star-by-star heliocentric radial velocities are outlined in Table 5. The value from Harris (1996, 2010 edition) catalogue is $-122.2 \mathrm{~km} \mathrm{~s}^{-1}$, with a central velocity dispersion of $1.3 \mathrm{~km} \mathrm{~s}^{-1}$. This result is also consistent with the value of $-123.2 \pm 1.0 \mathrm{~km} \mathrm{~s}^{-1}$ measured by Da Costa \& Seitzer (1989), where four giants were analysed, whose results were used by the authors to conclude that NGC 6366 has unambiguously halo kinematics. The authors used the $\left(V_{\mathrm{s}}, \Psi\right)$ plane model, where $V_{\mathrm{s}}$ is the radial velocity with respect to a stationary observer at the Sun and $\Psi$ is the angle between the line of sight to the cluster and the direction of Galactic rotation at the cluster (see Fig. 1 from Frenk \& White 1980, where the model is explained). Using low-resolution spectra of 14 giants, Dias et al. (2016) found a mean $\mathrm{RV}$ of $-137 \pm 54 \mathrm{~km} \mathrm{~s}^{-1}$. In their analysis, there are two objects in common with this work (stars 11 and 18) and the RVs for these individual objects agree in $<1 \mathrm{~km} \mathrm{~s}^{-1}$. Using the cross-correlation method, Johnson et al. (2016) found a mean heliocentric RV of $-122.3 \mathrm{~km} \mathrm{~s}^{-1}\left(\sigma=1.5 \mathrm{~km} \mathrm{~s}^{-1}\right)$ for NGC 6366. The proper motion analysis of Sariya \& Yadav (2015) gave zero membership probability for star 05 and membership probability $>0.95$ for stars 03 , 07,08 , and 11 . Stars 01,06 , and 11 were not present in their study. Based on our results for radial velocities, we assume cluster membership for all the eight stars, and the status of star 05 will be further discussed in the chemical analysis.

\subsection{Atmospheric parameters}

In Fig. 6 and Table 6 the photometric and spectroscopic effective temperatures are compared. The difference between the mean spectroscopic $T_{\text {eff }}$ and the mean $T_{\text {eff, }(V-K)}$ is $170 \mathrm{~K}(\sigma=89 \mathrm{~K})$. For the mean $T_{\text {eff, }(J-K)}$ the difference is $-108 \mathrm{~K}(\sigma=122 \mathrm{~K})$. We recall that our adopted uncertainty is $150 \mathrm{~K}$ (see Section 3.1). If spectroscopic and photometric measurements for individual stars are compared, the differences in $T_{\text {eff }}$ can be as big as $\sim 300 \mathrm{~K}$. As we adopted a fixed $E(B-V)$ value, the main reason for the discrepancy can 
Table 2. Atomic line list: wavelength, ionization state (integer part: atomic number; decimal part: .0 for neutral and .1 for single ionized species), atomic parameters $\chi$ and $\log g f$ and EWs for each star. This table is published in its entirety in the electronic edition of the paper. See the text for sources of the atomic parameters.

\begin{tabular}{lcccccccccrr}
\hline $\begin{array}{l}\text { Wavelength } \\
(\AA)\end{array}$ & Ion & $\begin{array}{c}\chi \\
(\mathrm{eV})\end{array}$ & $\log g f$ & $\begin{array}{c}01 \\
(\mathrm{~m} \AA)\end{array}$ & $\begin{array}{c}03 \\
(\mathrm{~m} \AA)\end{array}$ & $\begin{array}{c}05 \\
(\mathrm{~m} \AA)\end{array}$ & $\begin{array}{c}06 \\
(\mathrm{~m} \AA)\end{array}$ & $\begin{array}{c}07 \\
(\mathrm{~m} \AA)\end{array}$ & $\begin{array}{c}08 \\
(\mathrm{~m} \AA)\end{array}$ & $\begin{array}{c}11 \\
(\mathrm{~m} \AA)\end{array}$ & $\begin{array}{r}18 \\
(\mathrm{~m} \AA)\end{array}$ \\
\hline 6300.31 & 8.0 & 0.00 & -9.75 & 33.4 & 39.5 & 36.5 & 34.0 & 31.5 & 29.9 & 49.1 & 52.0 \\
6363.78 & 8.0 & 0.02 & -10.25 & 12.0 & 17.0 & 19.2 & 9.3 & 13.9 & 11.8 & $\ldots$ & 26.1 \\
5682.65 & 11.0 & 2.10 & -0.67 & 79.8 & $\ldots$ & 96.2 & 106.1 & $\ldots$ & 106.6 & 148.7 & 131.5 \\
5688.22 & 11.0 & 2.10 & -0.37 & 101.4 & $\ldots$ & 112.8 & 116.6 & $\ldots$ & 118.7 & 157.6 & 139.3 \\
6154.23 & 11.0 & 2.10 & -1.57 & 32.1 & 46.9 & 30.9 & 38.9 & 36.3 & 45.3 & 88.4 & 52.4 \\
6160.75 & 11.0 & 2.10 & -1.26 & 37.9 & 63.4 & 50.6 & 57.3 & 50.1 & 57.0 & 103.0 & 78.1 \\
$\ldots$ & $\ldots$ & $\ldots$ & $\ldots$ & $\ldots$ & $\ldots$ & $\ldots$ & $\ldots$ & $\ldots$ & $\ldots$ & $\ldots$ & $\ldots$ \\
\hline
\end{tabular}

Table 3. Line-by-line abundances obtained by the spectral synthesis method.

\begin{tabular}{lcccccccrr}
\hline$[\mathrm{X} / \mathrm{Fe}]$ & Wavelength $(\AA)$ & 01 & 03 & 05 & 06 & 07 & 08 & 11 & 18 \\
\hline$[\mathrm{Mn} / \mathrm{Fe}]$ & 6013.51 & -0.35 & -0.26 & -0.40 & -0.33 & -0.17 & -0.25 & -0.24 & -0.30 \\
{$[\mathrm{Mn} / \mathrm{Fe}]$} & 6016.64 & -0.31 & -0.18 & -0.36 & -0.37 & -0.18 & -0.31 & -0.40 & -0.33 \\
{$[\mathrm{Mn} / \mathrm{Fe}]$} & 6021.80 & -0.33 & -0.28 & -0.42 & -0.44 & -0.25 & -0.27 & -0.35 & -0.21 \\
{$[\mathrm{Y} / \mathrm{Fe}]$} & 5087.42 & -0.40 & $\ldots$ & -0.30 & -0.30 & $\ldots$ & -0.10 & -0.05 & -0.10 \\
{$[\mathrm{Y} / \mathrm{Fe}]$} & 5200.42 & -0.25 & $\ldots$ & -0.12 & -0.20 & $\ldots$ & -0.15 & -0.15 & 0.01 \\
{$[\mathrm{Ba} / \mathrm{Fe}]$} & 5853.69 & -0.22 & -0.04 & -0.15 & -0.20 & -0.06 & -0.01 & 0.00 & -0.18 \\
{$[\mathrm{Eu} / \mathrm{Fe}]$} & 6645.06 & 0.15 & 0.33 & 0.16 & 0.38 & 0.23 & 0.15 & 0.30 & 0.23 \\
\hline
\end{tabular}

be differential reddening. Both Campos et al. (2013) and Alonso et al. (1997) found that reddening increases from south to north in NGC 6366. If the two southernmost stars (03 and 11) are excluded, there is a trend between declination and the spectroscopicphotometric difference in both $(V-K)$ and $(J-K)$ calibrations. The spectroscopic-photometric difference increases from south to north, i.e. the photometric temperatures are 'colder' (redder) than they should be in the northernmost stars - if the spectroscopic temperatures are taken as 'true' $T_{\text {eff. }}$ The $\Delta \mathrm{E}(V-K)$ should be $\sim 0.5 \mathrm{mag}$ $-\Delta E(B-V) \sim 0.18$ mag using the relations given in Section $3-$ to account for this trend alone in the $(V-K)$ calibration, because an error of $0.01 \mathrm{mag}$ corresponds to a $5 \mathrm{~K}$ variation in the photometric $T_{\text {eff }}$ (Alonso et al. 1999), and the spectroscopic-photometric difference varies from $35 \mathrm{~K}$ (star 01) to $286 \mathrm{~K}$ (star 06). Concerning the $(J-K)$ calibration, if we assume the $23 \mathrm{~K}$ variation per $0.01 \mathrm{mag}$ given by Alonso et al. for stars with $(J-K)>0.5$, the $\Delta \mathrm{E}(J-K)$ should be $0.17 \mathrm{mag}$, or $\sim 0.3 \mathrm{mag}$ in $\Delta E(B-V)$, to account for the $\sim 400 \mathrm{~K}$ range in the spectroscopic-photometric difference in $T_{\text {eff }}-$ the difference is $-311 \mathrm{~K}$ for star 01 and $87 \mathrm{~K}$ in star 06. However, the HB stars have $(J-K) \sim 0.5$, and, for those bluer than this threshold, the variation in $T_{\text {eff }}$ per 0.01 mag given by Alonso et al. is $69 \mathrm{~K}$, resulting in $\Delta E(B-V) \sim 0.11 \mathrm{mag}$. A detailed reddening map of the entire area of the GC would be useful to clarify this issue, since the map from Alonso et al. (1997) is a rough estimate and the map from Campos et al. (2013), the most detailed to date, covers only a small central field. The region mapped in Campos et al. shows a $\Delta E(B-V)$ of 0.12 mag, indicating that our hypothesis of differential reddening as the main reason for the discrepancy in photometric and spectroscopic temperatures is feasible. Besides the differential reddening, there are the 'shifts' represented by the differences between mean photometric and mean spectroscopic temperatures, mentioned earlier in this paragraph, but those values - comparable to the adopted uncertainties - may be effect of using a unique value of $E(B-V)$ for all stars, if our hypothesis of differential reddening influence is correct.
The $\log g$ values are typical of giant stars (see e.g. table 5 of Alves-Brito et al. 2010). The difference of $\sim 0.9$ dex between HB stars and the two most luminous ones - those of the ESPaDOnS subsample - is expected. Star 03 has an intermediary value of $\log g=2.1$.

The microturbulent velocity is defined as a non-thermal component of the gas velocity on a smaller scale than the radial component of the line-forming region (Cantiello et al. 2009), being responsible for a portion of line broadening. $\xi_{\mathrm{t}}$ values were derived only spectroscopically. The first guess value was arbitrarily set as $1.0 \mathrm{~km} \mathrm{~s}^{-1}$, and the final values found are typical for giants (see also Alves-Brito et al. 2010, table 5).

As for metallicities, we found a mean $[\mathrm{Fe} / \mathrm{H}]=-0.60$ for NGC 6366, with a median $[\mathrm{Fe} / \mathrm{H}]=-0.62$. The mean metallicity is consistent with that found by Johnson et al. (2016) as well as studies based upon low-resolution data and photometry, mentioned in Section 1. The two subsamples analysed in this work do not present different trends in metallicity. The mean uncertainty $\left\langle\sigma_{*}\right\rangle$ found for $[\mathrm{Fe} / \mathrm{H}]$ is 0.20 dex, while the standard deviation of star-to-star variation $\sigma_{\mathrm{cl}}$ is $0.08 \mathrm{dex}$. All $[\mathrm{Fe} / \mathrm{H}]$ values are inside $1-\left\langle\sigma_{*}\right\rangle$ from the mean $[\mathrm{Fe} / \mathrm{H}]$. No metallicity variation was detected beyond uncertainties.

\subsection{Alpha elements}

As discussed in McWilliam (2016), the $\alpha$ elements analysed in this work $(\mathrm{O}, \mathrm{Mg}, \mathrm{Si}, \mathrm{Ca}$, and $\mathrm{Ti})$ are grouped under the ' $\alpha$ ' label because it was thought that they were produced by successive addition of $\alpha$ particles. However, more recent nucleosynthesis models for massive stars point to different channels of production for these elements, with only $\mathrm{O}$ having the $\alpha$ process as the main channel, while $\mathrm{C}$ and $\mathrm{Ne}$ hydrostatic burning dominate for $\mathrm{Mg}$ production. Explosive $\mathrm{O}$ burning is responsible for most $\mathrm{Si}$ and $\mathrm{Ca}$ abundances, while $\mathrm{Ti}$ is made in a variety of sites - with the most abundant isotope, ${ }^{48} \mathrm{Ti}$ being produced by (explosive) incomplete Si burning and, to a lesser extent, type Ia Supernovae (SNe) (e.g. Woosley \& Weaver 1995; 
Table 4. Sensitivities to $1 \sigma$ variation of the atmospheric parameters in the abundance ratios in stars 01 and 18. See the text for uncertainties in the atmospheric parameters.

\begin{tabular}{|c|c|c|c|c|}
\hline Element & $\begin{array}{c}\Delta T_{\text {eff }} \\
\pm 150 \\
(\mathrm{~K})\end{array}$ & $\begin{array}{l}\Delta \log g \\
\pm 0.3 \\
(\operatorname{dex})\end{array}$ & $\begin{array}{c}\Delta[\mathrm{Fe} / \mathrm{H}] \\
\quad \pm 0.1 \\
\quad(\mathrm{dex})\end{array}$ & $\begin{array}{c}\Delta \xi_{t} \\
\pm 0.2 \\
\left(\mathrm{~km} \mathrm{~s}^{-1}\right)\end{array}$ \\
\hline & & 01 & & \\
\hline $\mathrm{Fe}_{\mathrm{I}}$ & 0.15 & $<0.01$ & $<0.01$ & 0.05 \\
\hline $\mathrm{Fe}_{\text {II }}$ & 0.06 & 0.14 & 0.02 & 0.05 \\
\hline $\mathrm{O}_{\mathrm{I}}$ & 0.04 & 0.13 & 0.03 & $<0.01$ \\
\hline $\mathrm{NaI}$ & 0.11 & 0.02 & $<0.01$ & 0.03 \\
\hline $\mathrm{Mg}_{\mathrm{I}}$ & 0.11 & 0.01 & $<0.01$ & 0.04 \\
\hline $\mathrm{Al} \mathrm{I}_{\mathrm{I}}$ & 0.10 & $<0.01$ & $<0.01$ & $<0.01$ \\
\hline Si I & 0.05 & 0.03 & $<0.01$ & 0.02 \\
\hline $\mathrm{Ca} \mathrm{I}$ & 0.14 & 0.02 & $<0.01$ & 0.06 \\
\hline Sc II & $<0.01$ & 0.12 & 0.03 & 0.06 \\
\hline Ti I & 0.21 & $<0.01$ & $<0.01$ & 0.03 \\
\hline Ti II & $<0.01$ & 0.12 & 0.02 & 0.09 \\
\hline $\mathrm{V}_{\mathrm{I}}$ & 0.24 & $<0.01$ & $<0.01$ & 0.02 \\
\hline $\mathrm{Cr}_{\mathrm{I}}$ & 0.18 & 0.01 & $<0.01$ & 0.06 \\
\hline Mn I & 0.17 & $<0.01$ & 0.10 & 0.01 \\
\hline Cor & 0.15 & 0.01 & $<0.01$ & 0.02 \\
\hline $\mathrm{Ni}_{\mathrm{I}}$ & 0.15 & 0.01 & $<0.01$ & 0.04 \\
\hline $\mathrm{Y}_{\mathrm{II}}$ & 0.06 & 0.10 & 0.11 & 0.08 \\
\hline $\mathrm{Ba}$ II & 0.09 & 0.15 & 0.03 & 0.08 \\
\hline \multirow[t]{2}{*}{ Eu II } & 0.05 & 0.23 & $<0.01$ & 0.05 \\
\hline & & 18 & & \\
\hline $\mathrm{Fe}_{\mathrm{I}}$ & 0.11 & 0.04 & $<0.01$ & 0.06 \\
\hline $\mathrm{Fe}_{\text {II }}$ & 0.15 & 0.17 & 0.03 & 0.04 \\
\hline $\mathrm{O}_{\mathrm{I}}$ & 0.02 & 0.13 & 0.04 & $<0.01$ \\
\hline $\mathrm{Na} I$ & 0.14 & 0.02 & $<0.01$ & 0.05 \\
\hline $\mathrm{Mg}_{\mathrm{I}}$ & 0.09 & $<0.01$ & $<0.01$ & 0.06 \\
\hline $\mathrm{Al}$ I & 0.11 & $<0.01$ & $<0.01$ & 0.02 \\
\hline Si I & 0.05 & 0.07 & 0.01 & 0.03 \\
\hline $\mathrm{CaI}$ & 0.16 & 0.01 & $<0.01$ & 0.09 \\
\hline $\mathrm{Sc}_{\text {II }}$ & 0.02 & 0.13 & 0.03 & 0.08 \\
\hline Ti I & 0.23 & $<0.01$ & $<0.01$ & 0.06 \\
\hline Ti II & 0.03 & 0.13 & 0.03 & 0.09 \\
\hline $\mathrm{V}_{\mathrm{I}}$ & 0.28 & 0.02 & $<0.01$ & 0.09 \\
\hline $\mathrm{Cr} I$ & 0.16 & $<0.01$ & $<0.01$ & 0.06 \\
\hline Mn I & 0.18 & 0.03 & 0.09 & 0.04 \\
\hline CoI & 0.10 & 0.05 & 0.02 & 0.04 \\
\hline $\mathrm{Ni}$ I & 0.08 & 0.06 & 0.02 & 0.07 \\
\hline $\mathrm{Y}_{\mathrm{II}}$ & 0.02 & 0.10 & 0.08 & 0.12 \\
\hline Ba II & 0.04 & 0.11 & 0.05 & 0.12 \\
\hline Eu II & 0.03 & 0.15 & 0.05 & 0.02 \\
\hline
\end{tabular}

Table 5. Kinematic results.

\begin{tabular}{cccc}
\hline ID & $\begin{array}{c}\mathrm{RV} \\
\left(\mathrm{km} \mathrm{s}^{-1}\right)\end{array}$ & $\begin{array}{c}\bar{\sigma} \mathrm{RV} \\
\left(\mathrm{km} \mathrm{s}^{-1}\right)\end{array}$ & $\begin{array}{c}\text { \# of lines } \\
\text { measured }\end{array}$ \\
\hline 01 & -121.46 & 0.07 & 69 \\
03 & -119.23 & 0.05 & 69 \\
05 & -119.60 & 0.08 & 69 \\
06 & -119.06 & 0.07 & 69 \\
07 & -120.83 & 0.08 & 69 \\
08 & -125.17 & 0.06 & 69 \\
11 & -122.06 & 0.06 & 217 \\
18 & -121.80 & 0.05 & 217 \\
\hline
\end{tabular}

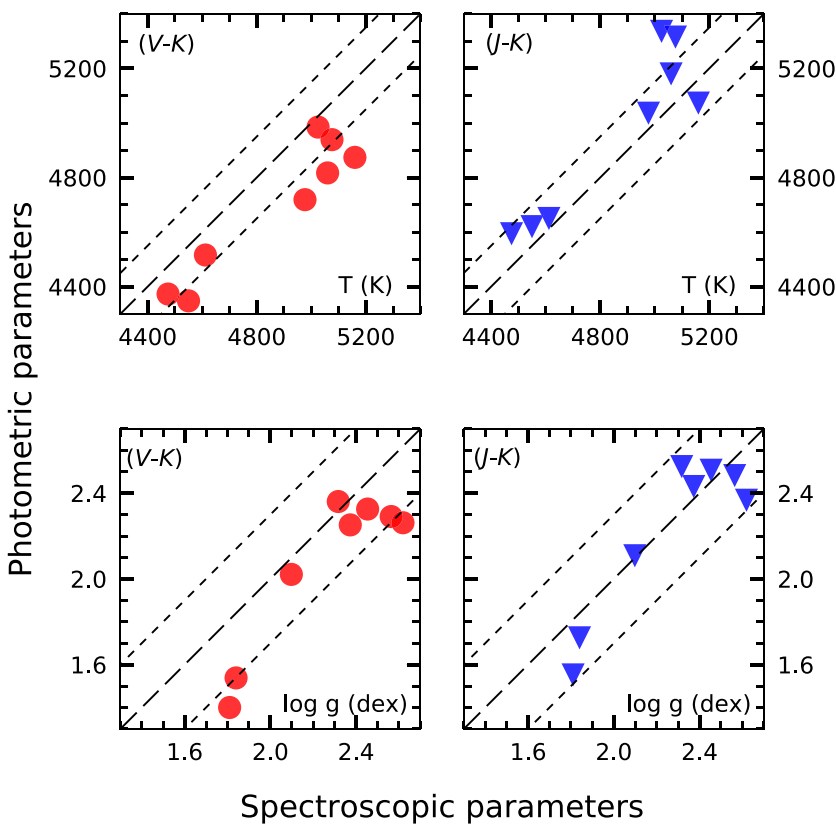

Figure 6. Comparison between atmospheric parameters obtained by spectroscopic ( $x$-axis) and photometric ( $y$-axis) methods. Red circles: photometric parameters derived from the $(V-K)$ calibration. Blue triangles: photometric parameters derived from the $(J-K)$ calibration. Dashed lines: identity function. Dotted lines: limits of the spectroscopic uncertainties adopted in this work (see Section 2).

Woosley, Heger \& Weaver 2002; Nomoto, Kobayashi \& Tominaga 2013). Since these elements are released to the interstellar medium by massive stars, the $[\alpha / \mathrm{Fe}]$ ratio is a tracer of the IMF of the primordial gas that formed a stellar population (see e.g. McWilliam 1997).

The mean $[\mathrm{O} / \mathrm{Fe}]$ for NGC 6366 is 0.48 dex (see Table 7 for a full list of chemical abundances and the standard deviations of each chemical abundance). Except for two outliers (stars 05 and 11) the individual $[\mathrm{O} / \mathrm{Fe}]$ deviates less than 0.05 dex from the mean value. The oxygen lines measured were the forbidden [OI] 6300.31 and $6363.78 \AA$. It was not possible to measure the $6363 \AA$ feature in star 11 , but its $\mathrm{O}$ abundance result seems not to be affected by this (see discussion about the $\mathrm{Na}-\mathrm{O}$ anticorrelation in Section 4.5).

For $[\mathrm{Mg} / \mathrm{Fe}]$ we found a mean value of $0.24 \mathrm{dex}, \sim 0.15$ dex more metal-poor than the bulge field plateau estimated by McWilliam (2016). Stars 03 and 07 lack the blue part of their spectra, thus only the $6318.71 \AA$ line was measured for all targets, while the $5711 \AA$ feature was measured for the other six stars, without star-to-star variation beyond mean uncertainties. The spread found in our sample overlaps those of both bulge and metal-rich halo field stars selected for comparison, as shown in Fig. 7.

With a longer line list for $\mathrm{Si}$ in comparison with $\mathrm{Mg}$, we measured $\langle[\mathrm{Si} / \mathrm{Fe}]\rangle=0.29$ for our sample. The $\mathrm{Si}$ abundances found in this work agree with both bulge and metal-rich halo field abundances, being on the high- $\alpha$ plateau.

The mean $[\mathrm{Ca} / \mathrm{Fe}]$ is $0.10 \mathrm{dex}$, with six stars having a $[\mathrm{Ca} / \mathrm{Fe}]$ ratio compatible with the solar value, within the associated uncertainties. The star-to-star variations of the abundances are smaller than the uncertainties. The mean $[\mathrm{Ca} / \mathrm{Fe}]$ is 0.20 dex below that found in Johnson et al. (2016) for NGC 6366, which is comparable to the mean uncertainty for $\mathrm{Ca}$ in this work, 0.19 dex. Inspection of the $[\mathrm{Ca} / \mathrm{Fe}]-[\mathrm{Fe} / \mathrm{H}]$ plot in Fig. 7 shows that NGC 6366 is deficient in 
Table 6. Atmospheric parameters derived by photometric and spectroscopic methods.

\begin{tabular}{lcccccccc}
\hline \multicolumn{9}{c}{ Spectroscopy } \\
ID & $\begin{array}{c}T_{\text {eff }} \\
(\mathrm{K})\end{array}$ & $\begin{array}{l}\log g \\
(\mathrm{dex})\end{array}$ & $\begin{array}{c}{[\mathrm{Fe} / \mathrm{H}]} \\
(\mathrm{dex})\end{array}$ & $\begin{array}{c}\xi_{\mathrm{t}} \\
\left(\mathrm{km} \mathrm{s}^{-1}\right)\end{array}$ & $\begin{array}{c}T_{\mathrm{eff},(V-K)}(\mathrm{K}) \\
\log g_{(V-K)}\end{array}$ & $\begin{array}{c}T_{\text {eff, }(J-K)}(\mathrm{dex}) \\
(\mathrm{K})\end{array}$ & $\begin{array}{c}\log g_{(J-K)} \\
(\mathrm{dex})\end{array}$ \\
\hline 01 & 5025 & 2.3 & -0.64 & 1.7 & 4985 & 2.4 & 5336 & 2.5 \\
03 & 4612 & 2.1 & -0.68 & 1.5 & 4516 & 2.0 & 4650 & 2.1 \\
05 & 5060 & 2.6 & -0.47 & 1.7 & 4817 & 2.3 & 5180 & 2.5 \\
06 & 5160 & 2.6 & -0.49 & 2.0 & 4874 & 2.3 & 5073 & 2.4 \\
07 & 4977 & 2.4 & -0.64 & 1.6 & 4719 & 2.3 & 5036 & 2.4 \\
08 & 5076 & 2.5 & -0.66 & 1.7 & 4939 & 2.3 & 5315 & 2.5 \\
11 & 4475 & 1.8 & -0.60 & 1.9 & 4373 & 1.4 & 4594 & 1.6 \\
18 & 4550 & 1.8 & -0.59 & 1.9 & 4348 & 1.5 & 4620 & 1.7 \\
\hline
\end{tabular}

Table 7. Chemical abundances (columns 2-9), in dex - where the solar photospheric abundances are from Asplund et al. (2009) - the mean abundance of each element (column 10), its mean uncertainties (column 11), standard deviations of star-to-star variation (column 12) and the ratio $\rho=\sigma_{\mathrm{cl}} /\left\langle\sigma_{*}\right\rangle$ in the last column.

\begin{tabular}{|c|c|c|c|c|c|c|c|c|c|c|c|c|}
\hline$[\mathrm{X} / \mathrm{Fe}]$ & 01 & 03 & 05 & 06 & 07 & 08 & 11 & 18 & Mean & $\left\langle\sigma_{*}\right\rangle$ & $\sigma_{\mathrm{cl}}$ & $\rho$ \\
\hline $\mathrm{O}_{\text {I }}$ & 0.46 & 0.47 & 0.61 & 0.47 & 0.50 & 0.52 & 0.35 & 0.47 & 0.48 & 0.15 & 0.07 & 0.47 \\
\hline $\mathrm{Na}$ I & 0.12 & 0.20 & 0.10 & 0.27 & 0.23 & 0.48 & 0.51 & 0.22 & 0.27 & 0.15 & 0.15 & 1.00 \\
\hline $\mathrm{Mg}_{\mathrm{I}}$ & 0.28 & 0.16 & 0.23 & 0.16 & 0.18 & 0.31 & 0.26 & 0.33 & 0.24 & 0.15 & 0.07 & 0.47 \\
\hline $\mathrm{Al}_{\mathrm{I}}$ & 0.31 & 0.44 & 0.34 & 0.32 & 0.40 & 0.39 & 0.29 & 0.41 & 0.36 & 0.13 & 0.05 & 0.38 \\
\hline Si I & 0.23 & 0.34 & 0.20 & 0.20 & 0.32 & 0.38 & 0.38 & 0.28 & 0.29 & 0.12 & 0.08 & 0.67 \\
\hline $\mathrm{Ca} I$ & -0.02 & 0.23 & 0.01 & 0.03 & 0.29 & 0.14 & 0.07 & 0.08 & 0.10 & 0.19 & 0.11 & 0.58 \\
\hline $\mathrm{Sc}_{\text {II }}$ & -0.14 & 0.12 & 0.10 & -0.02 & 0.24 & 0.10 & 0.08 & 0.11 & 0.08 & 0.17 & 0.11 & 0.65 \\
\hline Ti I & 0.19 & 0.33 & 0.24 & 0.33 & 0.33 & 0.28 & 0.23 & 0.25 & 0.27 & 0.24 & 0.05 & 0.21 \\
\hline Ti II & 0.28 & 0.38 & 0.24 & 0.34 & 0.33 & 0.48 & 0.37 & 0.28 & 0.34 & 0.18 & 0.08 & 0.44 \\
\hline $\mathrm{V}_{\mathrm{I}}$ & 0.01 & 0.14 & 0.05 & 0.07 & 0.08 & 0.15 & 0.27 & 0.24 & 0.13 & 0.28 & 0.09 & 0.32 \\
\hline Cr I & -0.05 & $\ldots$ & -0.09 & -0.03 & $\ldots$ & -0.07 & 0.02 & 0.01 & -0.04 & 0.16 & 0.04 & 0.25 \\
\hline Mn I & -0.33 & -0.24 & -0.39 & -0.38 & -0.20 & -0.28 & -0.33 & -0.28 & -0.30 & 0.20 & 0.07 & 0.35 \\
\hline CoI & 0.05 & 0.07 & -0.04 & 0.02 & 0.13 & 0.07 & 0.18 & 0.16 & 0.08 & 0.16 & 0.07 & 0.44 \\
\hline $\mathrm{Ni}$ I & 0.01 & 0.06 & -0.03 & 0.03 & 0.09 & 0.02 & 0.05 & 0.02 & 0.03 & 0.19 & 0.03 & 0.16 \\
\hline $\mathrm{Y}_{\mathrm{II}}$ & -0.33 & $\ldots$ & -0.21 & -0.25 & $\ldots$ & -0.13 & -0.10 & -0.05 & -0.18 & 0.18 & 0.10 & 0.56 \\
\hline Ba II & -0.22 & -0.04 & -0.15 & -0.20 & -0.06 & -0.01 & 0.00 & -0.18 & -0.11 & 0.18 & 0.09 & 0.50 \\
\hline Eu II & 0.15 & 0.33 & 0.16 & 0.38 & 0.23 & 0.15 & 0.30 & 0.23 & 0.24 & 0.20 & 0.09 & 0.45 \\
\hline
\end{tabular}

Ca when compared to the halo and that it overlaps the bulge field abundances, but one should also note that the bulge field scatter of $\mathrm{Ca}$ in the plot is very large, $\sim 0.40$ dex.

Ti I and $\mathrm{Ti}$ II lines were measured, with $\langle[\mathrm{Ti} \mathrm{I} / \mathrm{Fe}]\rangle=0.27$ and $\langle[\mathrm{Ti} \mathrm{II} / \mathrm{Fe}]\rangle=0.34$, differing by less than $0.10 \mathrm{dex}$. The resulting mean from both species is $[\mathrm{Ti} / \mathrm{Fe}]=0.31$. None of the Ti ions has $\rho>1$, and the bigger $\rho$ value for Ti II arises from its smaller mean uncertainty. When comparing with Alves-Brito et al. (2010) bulge field giants, Ti abundances in NGC 6366 are on the higher limit of the spread for its $[\mathrm{Fe} / \mathrm{H}]$ range, very close to the values calculated for 47 Tuc by Alves-Brito et al. (2005). The Ti abundances from this work agree well with those from metal-rich halo field stars obtained by Nissen \& Schuster (2010), as seen in Fig. 7.

Our results for the $\alpha$ elements match those found by Johnson et al. for NGC 6366, with the abundance of $\mathrm{Ca}$ being a possible exception. The abundances match both bulge and halo field abundances selected for comparison, with, again, the exception of $\mathrm{Ca}$, which deviates from the halo pattern. We found similar abundances to those of Dias et al. (2016) for the stars in common with their work. For star 11 the $[\mathrm{Fe} / \mathrm{H}]$ and $[\mathrm{Mg} / \mathrm{Fe}]$ values differ by $\sim 0.05$ dex, and for star 18 they differ by $\sim 0.10$ dex.

The $\alpha$-enhancements are consistent with those of other GCs, and NGC 6366 lies on the high- $\alpha$ plateau, with $[\alpha / \mathrm{Fe}]=0.28$. Despite being metal-rich, the GC position in the $[\alpha / \mathrm{Fe}]-[\mathrm{Fe} / \mathrm{H}]$ space is indicative of a relatively fast star formation rate instead of any Type
Ia SNe enrichment of the previous generation of stellar population (as discussed, e.g. in McWilliam 1997).

\subsection{Iron peak elements}

Compared with other atomic species, the Iron peak elements are less explored in the context of chemical evolution of GCs. These elements are produced in massive stars, in hydrostatic and explosive conditions, but also in Type Ia SNe. In Type II SNe, V, Co, and $\mathrm{Ni}$ are generated from explosive nuclear burning in complete Si-burning regions, while $\mathrm{Cr}$ and $\mathrm{Mn}$ are mainly synthesized in less deep incomplete Si-burning regions (Nomoto et al. 2013). For $\mathrm{Mn}$, Cescutti et al. (2008) suggest the use of metallicity-dependent yields for both types of SNe to reproduce observations in different populations. However, Kobayashi \& Nomoto (2009) argue that the $\mathrm{Mn}$ trend is caused by delayed enrichment of Type Ia SNe. The contribution of Type Ia SNe enhances $\mathrm{Mn}$ in high metallicity regimes, and this element can be useful to track the different astrophysical sites of nucleosynthesis (Kobayashi et al. 2006).

As for the $\alpha$ elements, none of the Fe-peak elements analysed in this work spreads beyond mean uncertainties, i.e. their $\rho$ is $\leq 1$. The mean [ $/ \mathrm{Fe}$ ] is 0.13 dex. $\mathrm{V}_{\mathrm{I}}$ is known for its extreme sensitivity to temperature variations in cold giants (as discussed in, e.g. Gratton et al. 2004). This affected the estimation of the uncertainties $\left(\left\langle\sigma_{*}\right\rangle\right.$ $=0.28$; see Table 4 for $T_{\text {eff }}$ sensitivities), but it did not translate into 


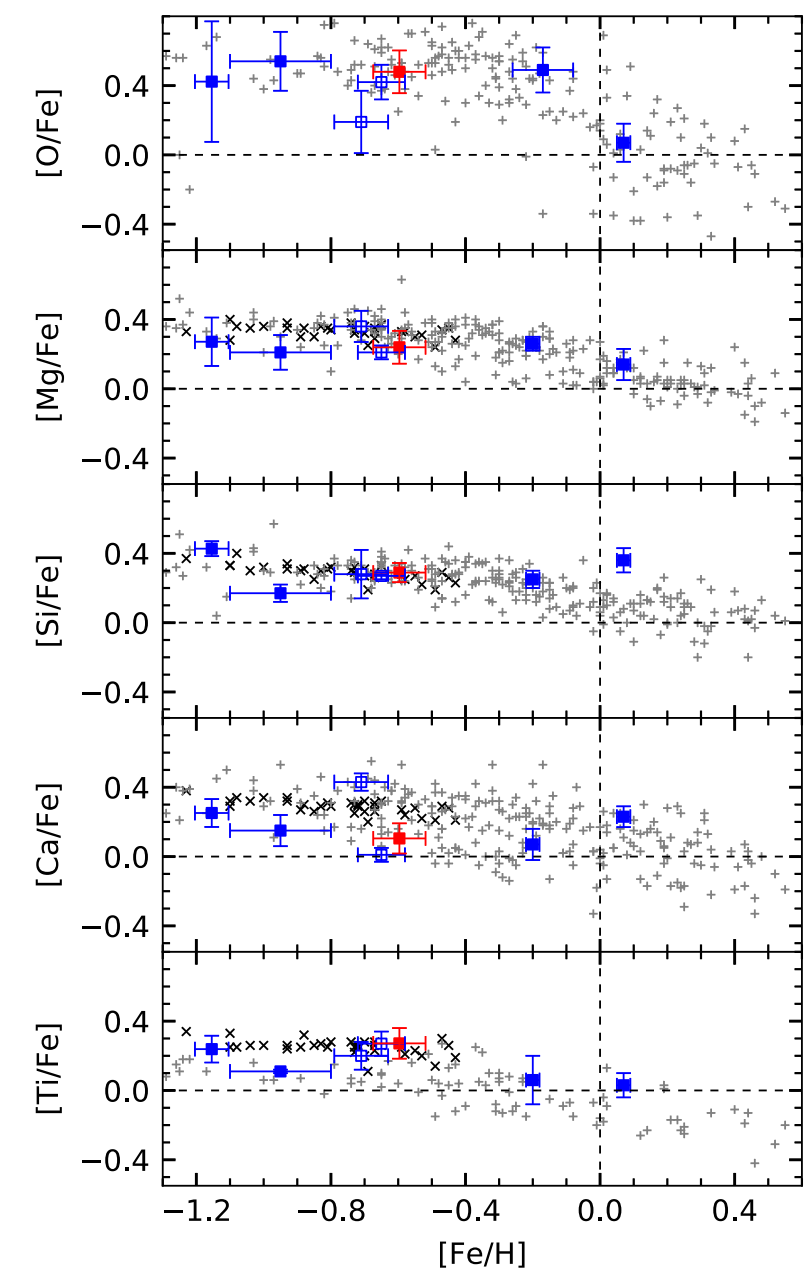

Figure 7. Comparsion of NGC $6366 \alpha$-elements abundance ratios with those of inner halo GCs, bulge field, and GCs stars. Red square: this work. Blue -filled squares: bulge GCs according to Bica et al. (2016). NGC 6266 (Yong et al. 2014), NGC 6522 (Barbuy et al. 2014), NGC 6553 (Alves-Brito et al. 2006), and NGC 6528 (Carretta et al. 2001). Blue open squares: metalrich GCs with $\mathrm{R}_{\mathrm{GC}}>4.5 \mathrm{kpc}$ (also according to Bica et al.), M 71 (Ramírez $\&$ Cohen 2002), and 47 Tuc (Alves-Brito et al. 2005). Grey crosses: bulge field stars (Alves-Brito et al. 2010; Johnson et al. 2014). Black ' $x$ ': 'high- $\alpha$ ' halo dwarfs from Nissen \& Schuster (2010). The error bars represent the star-to-star spread $(1 \sigma)$ of each element in a given GC. The dotted lines are the solar values. Both lists of GCs in this and other captions are ordered by $[\mathrm{Fe} / \mathrm{H}]$.

any spread. V shows some enhancement for most of the stars in the Baade's Window sample from McWilliam \& Rich (1994), but in most cases the abundances from McWilliam \& Rich and from this work overlap if we consider the uncertainties.

For the odd-Z element Mn we chose to synthesize the Mn I triplet at $6000 \AA$. The mean $[\mathrm{Mn} / \mathrm{Fe}]$ is $-0.30 \mathrm{dex}$, similar to the mean $[\mathrm{Mn} / \mathrm{Fe}]=-0.37$ of a sample of 19 GCs analysed by Sobeck et al. (2006). Inspecting Fig. 8, one can notice that NGC 6366 falls within the abundance spread of bulge field stars, but GCs, in general, follow a constant trend, independent from field stars in higher metallicities. Other bulge GCs shown in the plot also have similar deficient $[\mathrm{Mn} / \mathrm{Fe}]$, as do the most metal-rich $([\mathrm{Fe} / \mathrm{H}]>-1.5)$ halo stars. NGC 6366 behaves as a typical GC for Mn.

The $[\mathrm{Cr} / \mathrm{Fe}]$ of NGC 6366 is slightly below solar, with mean abundance of -0.03 dex, with $\sigma_{\mathrm{cl}}=0.04(N=6 \mathrm{stars})$. For $[\mathrm{Co} / \mathrm{Fe}]$, the mean is $0.08 \mathrm{dex}$, slightly above solar. As for $\mathrm{Cr}$, Co abundances

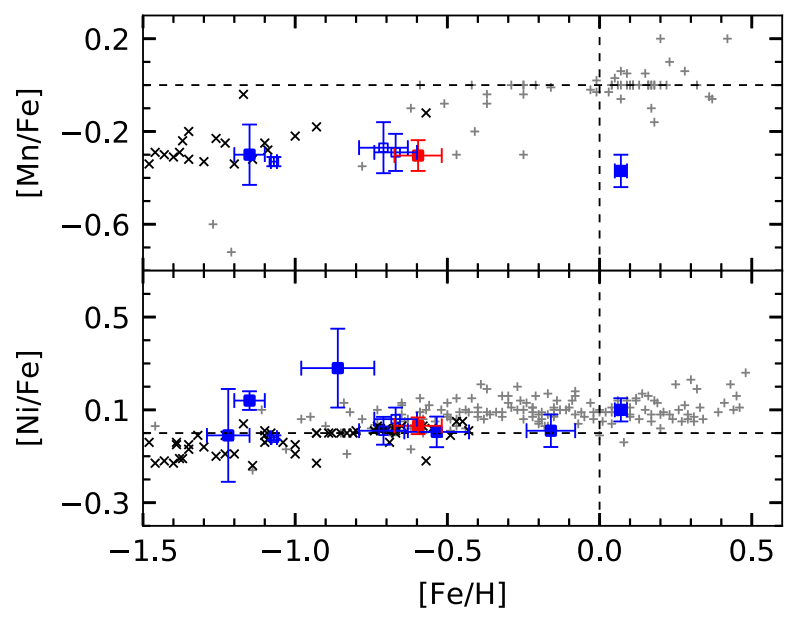

Figure 8. [Mn, Ni/Fe] versus $[\mathrm{Fe} / \mathrm{H}]$. The symbols are as in Fig. 7. bulge GCs: for Mn and Ni, NGC 6266 (Yong et al. 2014) and NGC 6528 (Carretta et al. 2001). For Ni, NGC 6723 (Gratton et al. 2015), Mercer 5 (Peñaloza et al. 2015), NGC 6342 (Johnson et al. 2016), and NGC 6553 (Cohen et al. 1999). metal-rich GCs with $R_{\mathrm{GC}}>4.5$ kpc: NGC 6362 (Massari et al. 2017), M 71 (Ramírez \& Cohen 2002), and 47 Tuc (Carretta et al. 2004). Field: bulge from Johnson et al. (2014) for $\mathrm{Ni}$, and from Barbuy et al. (2013) for Mn. Inner halo field (with probability >0.9) from Ishigaki, Aoki \& Chiba (2013) for Mn and Ni and 'high- $\alpha$ ' halo dwarfs from Nissen \& Schuster (2010, Ni only).

match those of the field (see e.g. fig. 11 of Johnson et al. 2014, for comparison for both $\mathrm{Cr}$ and $\mathrm{Co}$ with the bulge field and Nissen \& Schuster (2010) for Cr abundances in the halo field).

With several $(\sim 30)$ lines measured, our Ni measurements are solar. $[\mathrm{Ni} / \mathrm{Fe}]$ has an average of $0.03 \mathrm{dex}$ and the lowest starto-star spread $(\rho=0.16)$ of all the elements measured. In Johnson et al. (2016), the [Ni/Fe] of NGC 6366 is 0.10 dex and the difference between both studies is essentially due to the solar abundances adopted. In the metallicity range shown in Fig. 8, the $[\mathrm{Ni} / \mathrm{Fe}]$ values are between -0.20 and 0.20 dex for field stars, and most GCs shown fall in this interval - with NGC 6366 being very similar to GCs within its $[\mathrm{Fe} / \mathrm{H}]$ region.

\subsection{Light odd-Z elements}

As far as the internal GC chemistry is concerned, the light odd$\mathrm{Z}$ elements $\mathrm{Na}$ and $\mathrm{Al}$ are produced from proton captures in the $\mathrm{NeNa}$ and $\mathrm{MgAl}$ cycles, respectively. The $\mathrm{NeNa}$ cycle occurs in the same site, hence the same temperature, where $\mathrm{N}$ is produced from $\mathrm{O}$ via the $\mathrm{CNO}$ cycle, while the temperature needed for the $\mathrm{MgAl}$ process is higher than the temperature required for Na production at the progenitor (Denisenkov \& Denisenkova 1990; Langer, Hoffman \& Sneden 1993; Salaris, Cassisi \& Weiss 2002). As discussed in Section 1, there is no consensus about the nature of the polluters which might generate the $\mathrm{Na}-\mathrm{O}$ and $\mathrm{Mg}-\mathrm{Al}$ anticorrelations found in many GCs.

For $[\mathrm{Na} / \mathrm{Fe}]$ we found an average of $0.27 \mathrm{dex}$, with the largest spread for our sample $\left(\sigma_{\mathrm{cl}}=0.15\right)$, comparable to the mean uncertainties (ratio $\rho=1$ ). This element has usually a large spread in GCs, and our results are similar to those of Johnson et al. (2016) for this GC. For $\mathrm{Al}$, we have found an average abundance of $[\mathrm{Al} / \mathrm{Fe}]=$ 0.36 , an enhancement in agreement with the $\alpha$-like behaviour discussed by McWilliam (2016). In contrast with the possible $[\mathrm{Na} / \mathrm{Fe}]$ variation, $[\mathrm{Al} / \mathrm{Fe}]$ has $\rho=0.38$, thus no star-to-star variation was detected for this element. 

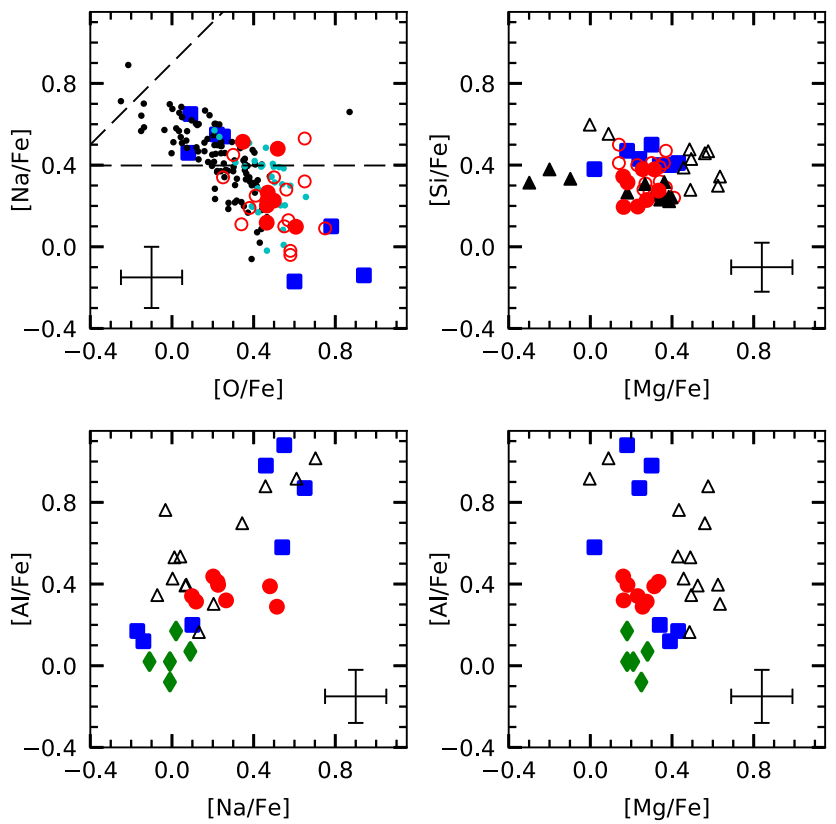

Figure 9. $[\mathrm{X} / \mathrm{Fe}]$ abundance ratios for light elements. Red -filled circles: this work. Red open circles: NGC 6366 from Johnson et al. (2016). Blue squares: NGC 6266 (Yong et al. 2014). Green diamonds: NGC 6558 (Barbuy et al. 2007). Black dots: 47 Tuc (Alves-Brito et al. 2005; Carretta et al. 2009a). Cyan dots: M 71 (Carretta et al. 2009a). Black-filled triangles: NGC 2808 and Black open triangles: NGC 7078 (Carretta et al. 2009b). The error bars are typical for this work. The dashed lines are the borders between P, I, and E components (see the text for discussion).

Fig. 9 shows correlation/anticorrelation pairs of some of the light elements. The $\mathrm{Na}-\mathrm{O}$ anticorrelation seems to be present in NGC 6366, and its extension is small. In this context, Carretta (2006) suggested the InterQuartile Range (IQR) as a quantitative measurement for the $\mathrm{Na}-\mathrm{O}$ anticorrelation, because of its low sensitivity to outliers. Carretta et al. (2010) describe, among other global parameters relations, an anticorrelated relation between the IQR of $[\mathrm{O} / \mathrm{Na}]$ and the absolute magnitudes of the GCs, a proxy measurement for their masses. According to Harris (1996, 2010 edition), NGC 6366 is one of the faintest GCs, with $M_{V}=-5.74$. Two other GCs present in the Na versus O plot, NGC 6266 and 47 Tuc, are among the most luminous Galactic GCs, and they have more extended anticorrelations. The IQR of NGC 6366 in $[\mathrm{O} / \mathrm{Na}]$ is 0.13 dex with our eight-star sample, while, e.g. Yong et al. (2014) calculated 1.11 for NGC 6266. Using data from the other highresolution study of NGC 6366 (Johnson et al. 2016), we calculated an IQR of 0.28 dex for [O/Na] for a sample of 13 stars. M71 (NGC 6838), another faint GC $\left(M_{V}=-5.61\right)$, which is also shown in Fig. 9, has [O/Na] IQR of 0.26. Carretta et al. argue from evidence given in Elsner et al. (2008) that M71 lost a higher-than-average fraction of its original mass, and estimate a 'true' $M_{V}$ of -7.2 mag. Since both NGC 6366 and M 71 share similar values for mass, IQR of $\mathrm{Na}-\mathrm{O}$ anticorrelation, metallicity, and evidence of strong massloss in the past (as mentioned for NGC 6366 in Section 1), we can take M71 as a comparative GC.

In our sample, only two stars - 08 and 11 - are above the border of the P ('primordial') and I ('intermediary') components, as defined in Carretta et al. (2009a). Of those, only star 11 has subsolar [O/Na] $=-0.16 \mathrm{dex}$, but still far from the threshold that characterizes the $\mathrm{E}$ ('extreme') population, $[\mathrm{O} / \mathrm{Na}]<-0.90$. The border between $\mathrm{P}$ and I components, which varies with metallicity (e.g. Carretta et al. 2010), was assumed at $[\mathrm{Na} / \mathrm{Fe}]=0.4$ in NGC 6366, the same value given for $47 \mathrm{Tuc}$, since both GCs have similar [Fe/H]. Membership of the $\mathrm{E}$ component can be ruled out for all the stars by visual inspection of Fig. 9. The aforementioned stars 08 and 11 are inside the I component, but their $(1 \sigma)$ error bars cross the P/I border. If we adopt a criterion that the measurements need to be located at more than $1 \sigma$ from the border between components for a bona fide characterization, star 18 also does not have a defined component and star 06 barely passes the requirement for the primordial population.

If we ignore the error bars, the fractions of $\mathrm{P}$ and I components found in this work are 0.75 and 0.25 , respectively. However, no relations between GC parameters outlined in Carretta et al. (2010, figs 11-16) involving the $\mathrm{P} / \mathrm{I} / \mathrm{E}$ components can reproduce our results. Our values of $[(\mathrm{Mg}+\mathrm{Al}+\mathrm{Si}) / \mathrm{Fe}]=0.30$ and $[\mathrm{Ca} / \mathrm{Fe}]=0.10$ would give a $\mathrm{P} / \mathrm{I}$ ratio $\geq 1$, but also a nonzero fraction of $\mathrm{E}$ stars. The (low) Na-O IQR found in this work matches the absence of the extreme component, but fails to match with the (high) $\mathrm{P}$ and (low) I fractions, since, according to Carretta et al., the $\mathrm{Na}-\mathrm{O}$ IQR is correlated with the former and anticorrelated with the later. If we use M71 as a proxy, the P/I/E fractions should be $\sim 0.28 / 0.72 / 0.00$ (Carretta et al. 2009a), i.e. the inverse found in our sample. However, for these $\mathrm{P} / \mathrm{I} / \mathrm{E}$ proportions, $[\mathrm{Ca} / \mathrm{Fe}]$ should be at least 0.25 dex $(\sim 1 \sigma$ higher, but similar to the value found in Johnson et al. (2016)), and $[(\mathrm{Mg}+\mathrm{Al}+\mathrm{Si}) / \mathrm{Fe}]$ should be at least 0.15 dex above the value derived in this work. Adopting the $1 \sigma$ criterion, half of our sample is primordial, still a high fraction if the sample is representative of the entire $G C$, which might not be the case because it has only eight stars. The $\mathrm{Na}-\mathrm{O}$ anticorrelation of NGC 6366 should be more extended if the GC has the estimated $\mathrm{P} / \mathrm{I}$ ratio, and an extreme population should be present (see fig. 11 of Carretta et al. (2010)), contradicting what is shown in Fig. 9.

Models of GC formation predict a homogeneous radial distribution after relaxation (D'Ercole et al. 2008; Decressin et al. 2010), with the SG being more concentrated in the centre of the GC in the earlier stages of dynamical evolution (Vesperini et al. 2013). Indeed, some GCs show a spatial correlation between the predominance of each stellar population and the distance to the centre of the GC (e.g. Bellini et al. 2009; Zoccali et al. 2009), but Larsen et al. (2015) have found an inverse trend in M 15, where giants of the first generation $(\mathrm{FG})$ are more concentrated in the centre. Using the ratio between age and current half-mass relaxation time $t / t_{\mathrm{rh}}$ to estimate the dynamical evolution of a GC, as suggested by Vesperini et al., we find $t / t_{\mathrm{rh}} \approx 20$ using the age from Campos et al. (2013) and $t_{\mathrm{rh}}$ from Harris (1996, 2010 edition). This value represents the stage when FG and SG should become well mixed, according to Fig. 6 of Vesperini et al. Therefore, no bias in the P/I/E (FG/SG) fractions due to a narrow radial distribution of the sample should be expected. Also, neither our sample nor that of Johnson et al. (2016), which has a broader radial distribution, presents any correlation between star positions and the $[\mathrm{O} / \mathrm{Na}]$ ratio.

As noted by Charbonnel (2016), the presence of multiple populations in star clusters is correlated with the high compactness index $\mathrm{C}_{5} \equiv\left(M_{c l} / 10^{5} \mathrm{M}_{\odot}\right) /\left(\mathrm{r}_{h} / \mathrm{pc}\right)$, where $M_{\mathrm{cl}}$ is the cluster mass and $r_{h}$ is the half-mass radius. Gas expulsion tends to be more difficult in more compact star clusters due to their deeper potential well. NGC 6366 is one of the least compact Galactic GCs, with $C_{5}=0.058$ (Krause et al. 2016, see also fig. 2 of Charbonnel) for an estimated half-mass radius of $5.05 \mathrm{pc}(\approx 5 \mathrm{arcmin})$. As the small [O/Na] IQR of NGC 6366 is well established from two different 
works, further studies of light elements with bigger samples and greater S/N ratio would be interesting to determine if NGC 6366 really lacks a second generation (SG) of stars. This is apparently the case, as suggested by chemical analyses done to date (this work; Johnson et al. (2016)) and to the GC global parameters such as mass - the least massive GC with clear FG/SG separation is NGC 6362 (Mucciarelli et al. 2016), which has $M_{V}=-6.95$.

As previously mentioned, this work presents the first determination of $\mathrm{Al}$ abundances in NGC 6366 using high-resolution spectra. While some GCs present a correlation between $\mathrm{Na}$ and $\mathrm{Al}$ (e.g. Carretta et al. 2009b), inspection of Fig. 9 reveals no such trend in NGC 6366. The absence of correlation is related to the lack of variation of $\mathrm{Al}$, a common feature in GCs more metal-rich than $[\mathrm{Fe} / \mathrm{H}]$ $\geq-1.25$ and also in the least massive ones (see e.g. Carretta et al. (2009b) and Cordero et al. (2015)). Comparing NGC 6366 with other GCs of similar metallicity, no Na-Al correlation is apparent in, e.g. M71 (Cordero et al. 2015) and the situation for 47 Tuc is unclear (see e.g. Carretta et al. 2013 and Cordero et al. 2014, for different results), but we should point out that 47 Tuc is a massive GC instead. As discussed in Cordero et al. (2015), such dependence in the star-to-star variation of $\mathrm{Al}$ with metallicity can be reproduced by the AGB pollution scenario, as shown in Ventura et al. (2014, fig. 2 ), which reproduces to a rough approximation the $[\mathrm{Al} / \mathrm{Fe}]$ spread in NGC 6366 and its median value and also the greater scatter in the more metal-poor regime. However, we should note that the derived abundances in their models are for SG/Na-enhanced stars, whose existence in NGC 6366 is not clear. In addition, leakage of the $\mathrm{MgAl}$ process in $\mathrm{Si}$, which is found in some GCs (see discussion in Gratton et al. 2012, and references therein), is not found for NGC 6366, in contrast with GCs like NGC 2808 and NGC 7078, as seen in the top right plot of Fig. 9.

\subsection{Neutron-capture elements}

Elements with atomic mass $A>56$ are synthesized by capture of neutrons by Fe-peak nuclei in a diversity of astrophysical sites (Karakas \& Lattanzio 2014). The main channels of neutron capture are the s-process and the r-process. The s-process ('slow') is characterized by neutron-capture occurring on a time-scale longer than that of $\beta$-decay, i.e. a $\beta$-emission will occur before the capture of another neutron by the nucleus. It is done in low-neutron-density environments $-\mathrm{N}_{n} \leq 10^{8}$ neutrons $\mathrm{cm}^{-1}$ (Karakas \& Lattanzio 2014). The He-burning core and convective C-burning shell (atomic mass up to A 90) of massive stars (e.g. Pignatari et al. 2010; Frischknecht, Hirschi \& Thielemann 2012; Frischknecht et al. 2016) and interiors of AGB stars (atomic mass above $A \sim 90$ ) (e.g. Bisterzo et al. $2011,2014,2017)$ are the main sites. In the solar neighbourhood, elements such as $\mathrm{Y}$ and $\mathrm{Ba}$ can be used as tracers of the s-process (Simmerer et al. 2004). The r-process ('rapid') differs from the s-process by having a rate of neutron-capture faster than the timescale of $\beta$-decay. Despite Type II SNe being traditionally suggested as the main site of this process, its place of origin is not well understood (Thielemann et al. 2011). Other proposed sites are collisions between neutron stars and black holes (Lattimer \& Schramm 1974) and neutron star mergers (e.g. Eichler et al. 1989; Freiburghaus, Rosswog \& Thielemann 1999; Rosswog et al. 2014). Recent observations give evidence to the collision/merger scenarios (Tanvir et al. 2013; Drout et al. 2017). Observationally, it is well known that Eu is a good tracer of the r-process in the solar neighbourhood (Simmerer et al. 2004).

The lightest neutron-capture element measured in this work, Y, has a mean abundance of $[\mathrm{Y} / \mathrm{Fe}]=-0.18$. It was not possible to infer Y abundances for Stars 03 and 07 because there were no lines available in their spectra. The mean $\mathrm{Y}$ abundance is similar to those of NGC 6388 (Carretta et al. 2007), NGC 6441 (Gratton et al. 2006), and M 71 (Ramírez \& Cohen 2002), all of them in the range $-0.80<[\mathrm{Fe} / \mathrm{H}]<-0.40$. The more metal-poor GCs NGC 6266 and NGC 6522 present enhanced [Y/Fe] values (Yong et al. 2014, and Barbuy et al. 2014, respectively). In the (very) metal-poor GC NGC 6397, Lind et al. (2011) found $[\mathrm{Y} / \mathrm{Fe}]=-0.26$, using the same Y II lines (5087 and $5200 \AA$ ) from this work. NGC 6397 was also studied by James et al. (2004), with Y abundances similar to those of Lind et al., and their mean [Y/Fe] for 47 Tuc subgiants is -0.11 dex. However, the results of this element for 47 Tuc must be interpreted with caution, since its $[\mathrm{Y} / \mathrm{Fe}]$ found in the literature range from -0.13 to 0.65 dex (e.g. Wylie et al. 2006; McWilliam \& Bernstein 2008). The differences could be due to different methods and line lists employed. Thygesen et al. (2014) attribute the possible overestimation of abundances in $\mathrm{Y}_{\text {II }}$ lines in some studies as due to their choice of not applying hyperfine structure treatment. In spite of that, the agreement between the works of James et al. and Lind et al., using different line lists, gives some confidence to our result, derived from the same lines used by Lind et al.

For $\mathrm{Ba}$, a mean abundance of $-0.11 \mathrm{dex}$ is found, with star-tostar spread of $\sigma=0.09$. Most stars are less than $1 \sigma$ from solar $[\mathrm{Ba} / \mathrm{Fe}]$. The line used to derive $\mathrm{Ba}$ abundances was the $5853 \AA$ feature, whose hyperfine and isotopic splitting is negligible (see e.g. Mashonkina \& Zhao 2006). The result deviates from the raising trend of $[\mathrm{Ba} / \mathrm{Fe}]$ with $[\mathrm{Fe} / \mathrm{H}]$ in Galactic GCs identified by D'Orazi et al. (2010), which analysed a homogeneous sample of more than $1200 \mathrm{GC}$ stars. In their analysis, $[\mathrm{Ba} / \mathrm{Fe}]$ reaches a peak of $\sim 0.60$ at $[\mathrm{Fe} / \mathrm{H}] \sim-1.10$, while the most metal-rich GCs of their sample, 47 Tuc and M71, seem to deviate, being less Ba-abundant than expected by a linear fit (see their Fig. 1). The [Ba/Fe] derived for NGC 6366 deviates even more from the raising trend, and falls in the $[\mathrm{Ba} / \mathrm{Fe}]$ range of the field stars with the same metallicity (see also fig. 6 of Van der Swaelmen et al. 2016). It should be noted that, except for the aforementioned 47 Tuc and M71, all GCs from D'Orazi et al. have $[\mathrm{Fe} / \mathrm{H}]<-1$. Barbuy et al. (2014) and Yong et al. (2014) measured enhanced $[\mathrm{Ba} / \mathrm{Fe}] \sim 0.35$ for the intermediate-metallicity GCs NGC 6522 and NGC 6266, respectively, with some agreement with the trend from D'Orazi et al. In the metal-rich range, AlvesBrito et al. (2006) found that NGC 6553 is depleted in this element, with mean $[\mathrm{Ba} / \mathrm{Fe}]=-0.28$, while Carretta et al. (2001) measured a mild enhancement of 0.14 dex for NGC 6528. As for Y, NGC 6366 falls in the group of GCs, which are deficient in Ba.

The abundance of r-process elements in Galactic GCs is enhanced w.r.t. to the Sun, with a few exceptions (see e.g. Gratton et al. 2004). For NGC 6366 we calculated $\langle[\mathrm{Eu} / \mathrm{Fe}]\rangle=0.24(\sigma=0.09)$, assuming solar isotopic ratios of ${ }^{151} \mathrm{Eu}$ and ${ }^{153} \mathrm{Eu}$ (Asplund et al. 2009, table $3)$. As seen in Fig. 10, the mean $[\mathrm{Eu} / \mathrm{Fe}]$ derived in this work lies in the bulge/thick-disc field range. Also, as the mean values of $[\mathrm{Eu} / \mathrm{Fe}]$ and $[\alpha / \mathrm{Fe}]$ are very similar in our sample, we suggest that, taking the uncertainties into account, both $\alpha$ - and r-process elements in NGC 6366 might have similar astrophysical origin.

Since neutron-capture elements are produced by both s- and rprocesses, it can be useful to calculate the ratio [s/r] between the abundance of elements whose production is dominated, respectively, by the s-process and the r-process. A higher (positive) [s/r] indicates dominance of nucleosynthesis of heavy elements in sites associated with the s-process, e.g, AGB stars, while a lower (negative) $[\mathrm{s} / \mathrm{r}]$ suggests predominance of sites associated with the $\mathrm{r}$ process, e.g. Type II SNe and/or neutron star mergers. We chose to use the Ba results as a proxy to the s-process history due to its 


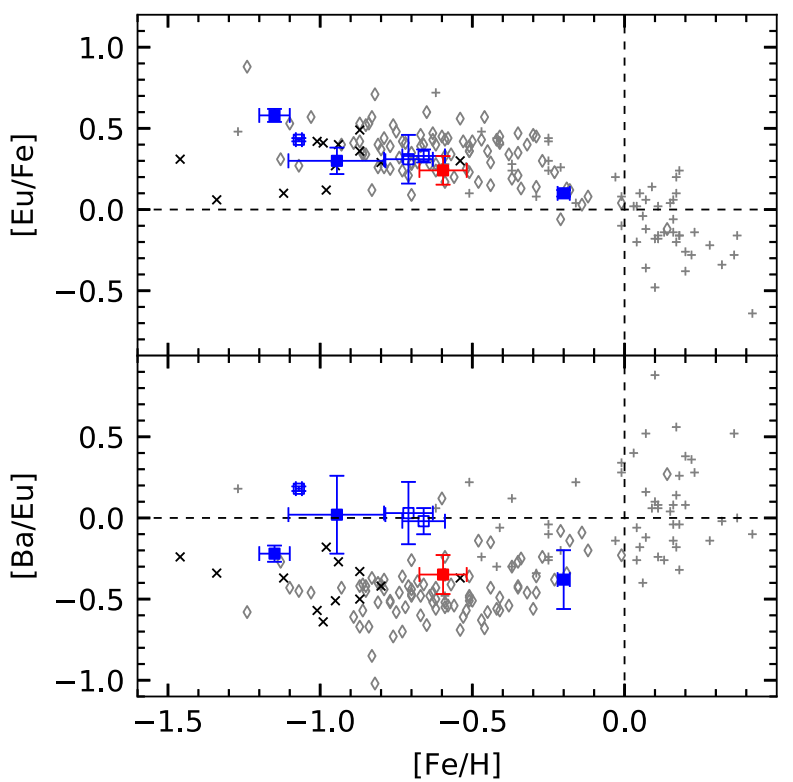

Figure 10. Top: $[\mathrm{Eu} / \mathrm{Fe}]$ versus $[\mathrm{Fe} / \mathrm{H}]$. Bottom: $[\mathrm{Ba} / \mathrm{Eu}]$ versus $[\mathrm{Fe} / \mathrm{H}]$. The symbols are as in Fig. 7, with grey open diamonds representing thick disc field stars. Bulge GCs: NGC 6266 (Yong et al. 2014), NGC 6522 (Barbuy et al. 2014), NGC 6553 (Alves-Brito et al. 2006). Metal-rich GCs with $R_{\mathrm{GC}}>4.5 \mathrm{kpc}$ : NGC 6362 (Massari et al. 2017), M 71 (Ramírez \& Cohen 2002), 47 Tuc (Alves-Brito et al. 2005). Field: bulge stars from Van der Swaelmen et al. (2016), thick disc and halo stars from Reddy, Lambert \& Allende Prieto (2006). The error bars represent the standard deviations of the GCs samples. The dotted lines are the solar values.

higher s-process proportion in the Solar system than $\mathrm{Y}$, while $\mathrm{Eu}$ is the r-process representative. For NGC 6366 we found $\langle[\mathrm{Ba} / \mathrm{Eu}]\rangle$ $=-0.35$. This ratio suggests some dominance of $\mathrm{r}$-process in the formation of heavy elements in this GC, since it is closer to the pure r-process solar ratio $[\mathrm{Ba} / \mathrm{Eu}]=-0.82$ than to the pure s-process solar ratio $[\mathrm{Ba} / \mathrm{Eu}]=1.60$ (both values derived from table 1 of Sneden, Cowan \& Gallino 2008). The [Ba/Eu] ratio also lies in the (mostly thick disc) field range, and is lower than those of the GCs of similar metallicities evaluated in Fig. 10. Summing up the $[\mathrm{Ba} / \mathrm{Eu}]$ value and the similarities between the $[\mathrm{Eu} / \mathrm{Fe}]$ and $[\alpha / \mathrm{Fe}]$ ratios, the primordial composition of NGC 6366 may have been previously enriched by objects with short time-scale, likely Type II SNe, with mild contribution of other sites like AGBs.

\subsection{Global abundance pattern}

We present in Fig. 11 the global abundance pattern for NGC 6366, with star 05 highlighted. As previously discussed in Section 4.1, Sariya \& Yadav (2015) gave zero membership probability to star 05 based on its proper motion, while our radial velocity measurements suggest a value compatible with NGC 6366 kinematics. Chemically, this star follows the general abundance pattern of the GC. Despite being the less-abundant star in some (but not all) Fe-peak elements, its deviation is negligible when compared to the uncertainties and does not contribute to any detectable star-to-star abundance spread. Also, star 05 presents a low-Na/high-O pattern that is typical of GCs stars.

Past studies noted similarities between NGC 6366 and 47 Tuc, such as their HB morphology, RR Lyrae population and metallicity (e.g. Sarajedini et al. 2007, and references therein). However, 47 Tuc

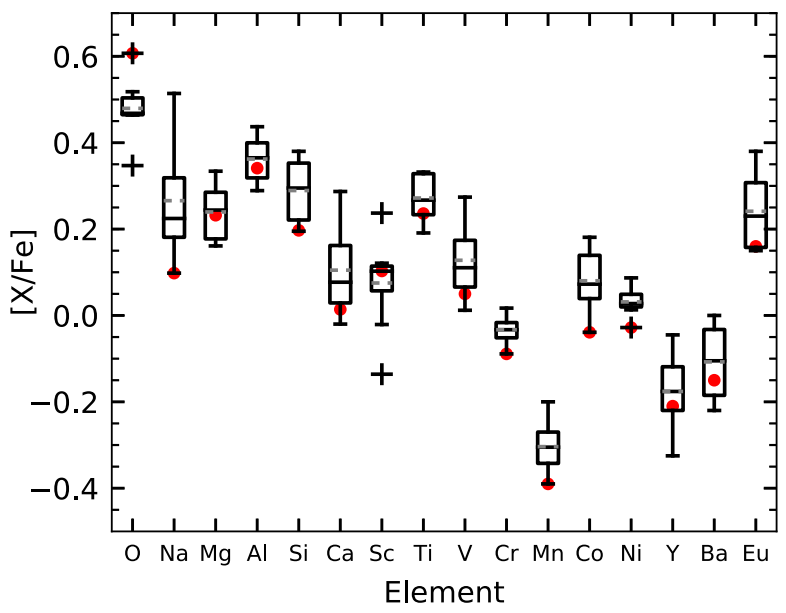

Figure 11. Star-to-star abundance spread for all elements measured in this work, except Fe. Boxes: the interquartile ranges (IQR). Whiskers: data inside $1.5 *$ IQR limits. Black horizontal lines: medians. Grey dashed lines: means. Crosses: outliers. Red circles: abundances of star 05.

is one of the most massive Galactic GCs, as opposed to NGC 6366, and it may have had a different dynamical history. Indeed, it has $t / t_{\mathrm{rh}} \approx 3$ (Harris 1996, 2010 edition; Cordero et al. 2015), much less than the estimation given for NGC 6366 in Section 4.5. Chemically, 47 Tuc, unlike NGC 6366, has a clear Na-O anticorrelation - even with the anticorrelation extension in 47 Tuc being smaller than what should be expected for its mass, the $\mathrm{Na}-\mathrm{O}$ anticorrelation is present in this GC. Also, as discussed in Section 4.5, it is not clear if its starto-star variation (or lack of thereof) in $\mathrm{Al}$ is similar to the result found in this work for NGC 6366. Of the s-process dominated elements evaluated here, 47 Tuc presents enhancement, while we show in this work that NGC 6366 is deficient - despite the Y measurements variation in 47 Tuc, as discussed in the previous section, its reported $[\mathrm{Ba} / \mathrm{Fe}]$ values are more consistent (e.g. Alves-Brito et al. 2005; D'Orazi et al. 2010; Thygesen et al. 2014).

On the other hand, NGC 6366 and M71 share similar global parameters, as discussed in Section 4.5. Chemically, both GCs have similar Na-O anticorrelations (see Fig. 9) and both lack star-to-star variation in Al. NGC 6366 and M 71 abundances only differ more than $\sim 0.15$ dex in $\mathrm{Ba}$, and possibly $\mathrm{Ca}$ - however, the $[\mathrm{Ca} / \mathrm{Fe}]$ from Ramírez \& Cohen (2002) shown in Fig. 7 may be overestimated, see e.g. the results of Meléndez \& Cohen (2009) and Mészáros et al. (2015) for M 71. Despite the difference in $\mathrm{Ba}$, the [Y/Fe] values of both clusters match, but a detailed comparison of both GCs in the sprocess family would clarify their level of similarity. They possibly share dynamical histories alike, due to their masses and past massloss. In this sense, a detailed analysis of NGC 6366 orbit, like that done to M 71 and other GCs in Dinescu, Girard \& van Altena (1999) would be useful. Judging by their chemical resemblance, NGC 6366 must have a low-eccentricity, low-inclination orbit, occupying the outer bulge/inner halo of the Milky Way. On the other hand, an orbit with higher eccentricity and low perigalactic distance could be a hint to a bulge origin.

In summary, NGC 6366 has a bulge-like metallicity, while both its kinematics and chemical similarity with M 71 and, possibly, 47 Tuc indicate otherwise. Also, a further homogeneous chemical analysis including NGC 6366 and these two GCs could help to improve our understanding of their internal chemistry and, maybe, clarify the situation of NGC 6366 in the Galactic context. 


\section{CONCLUSIONS}

Our analysis confirms NGC 6366 as a metal-rich GC, with no starto-star $[\mathrm{Fe} / \mathrm{H}]$ spread, based on eight giant stars. None of the elements measured present star-to-star variation greater than the uncertainties, i.e. the metallic content of NGC 6366 is very homogeneous.

Being considered a GC with halo kinematics, NGC 6366 has a chemical abundance pattern which can be consistent with both the bulge and the $\alpha$-rich, metal-rich halo. The $[\mathrm{Fe} / \mathrm{H}]$ abundance of -0.60 dex puts this GC in the metal-rich group of GCs, dominated by GCs associated with the bulge. However, it also presents similarities with the non-bulge GC M71, which orbits the Galaxy in the inner thick disc according to Dinescu et al. (1999). Given these similarities, NGC 6366 possibly has an orbit with low eccentricity close to the Galactic plane, but a detailed study of its orbital dynamics, preferentially with Gaia proper motion data, should be made before drawing conclusions. A resemblance with 47 Tuc is not evident chemically, but additional data from $\mathrm{Al}$ and the s-process elements in both GCs may be useful to clarify this point.

On the question of multiple populations, we have not found any unambiguous SG star. Based on the results of light odd- $Z$ abundances, it can be assumed that our entire sample, or at least most of it, is from the FG. When comparing this work with the analysis of Johnson et al. (2016), both samples overlap in Na-O diagram, showing a barely detectable, if any, $\mathrm{Na}-\mathrm{O}$ anticorrelation. Our study found no $\mathrm{Mg}-\mathrm{Al}$ anticorrelation and no star-to-star variation in $\mathrm{Al}$.

If NGC 6366 contains a simple stellar population, which is possibly the case, it enters the list of Galactic GCs without detection of multiple populations, adding evidence that, at least in the Galaxy, such phenomenon occurs only in GCs brighter than $M_{V} \approx-7$. Additional high-precision photometric data as well as studies focused upon high-resolution spectroscopy at optical and infrared wavelengths targeting larger samples and fainter magnitudes would help to improve our understanding of this very reddened GC.

\section{ACKNOWLEDGEMENTS}

We thank the anonymous referee for important comments and suggestions. Partial financial support for this research comes from BICUFRGS institutional program and $\mathrm{CNPq}$ (Brazil). This publication makes use of data products from the 2MASS, which is a joint project of the University of Massachusetts and the Infrared Processing and Analysis Center/California Institute of Technology, funded by the National Aeronautics and Space Administration and the National Science Foundation. The Digitized Sky Surveys were produced at the Space Telescope Science Institute under U.S. Government grant NAG W-2166. The images of these surveys are based on photographic data obtained using the Oschin Schmidt Telescope on Palomar Mountain and the UK Schmidt Telescope. IRAF is distributed by the National Optical Astronomy Observatories, which are operated by the Association of Universities for Research in Astronomy, Inc., under cooperative agreement with the National Science Foundation. Based on observations collected at the European Organization for Astronomical Research in the Southern hemisphere under ESO programme 69.B-0467. Based on observations obtained at the Canada France Hawaii Telescope, which is operated by the National Research Council of Canada, the Institut National des Sciences de IUnivers of the Centre National de la Recherche Scientique of France, and the University of Hawaii.

\section{REFERENCES}

Allen C. W., 1976, Astrophysical Quantities

Alonso A., Salaris M., Martinez-Roger C., Straniero O., Arribas S., 1997, A\&A, 323, 374

Alonso A., Arribas S., Martinez-Roger C., 1998, A\&AS, 131, 209

Alonso A., Arribas S., Martínez-Roger C., 1999, A\&AS, 140, 261

Alonso A., Arribas S., Martínez-Roger C., 2001, A\&A, 376, 1039

Alves-Brito A. et al., 2005, A\&A, 435, 657

Alves-Brito A. et al., 2006, A\&A, 460, 269

Alves-Brito A., Meléndez J., Asplund M., Ramírez I., Yong D., 2010, A\&A, 513, A35

Asplund M., Grevesse N., Sauval A. J., Scott P., 2009, ARA\&A, 47, 481

Barbuy B., Zoccali M., Ortolani S., Minniti D., Hill V., Renzini A., Bica E., Gómez A., 2007, AJ, 134, 1613

Barbuy B. et al., 2013, A\&A, 559, A5

Barbuy B. et al., 2014, A\&A, 570, A76

Bedin L. R., Piotto G., Anderson J., Cassisi S., King I. R., Momany Y., Carraro G., 2004, ApJ, 605, L125

Bellini A., Piotto G., Bedin L. R., King I. R., Anderson J., Milone A. P., Momany Y., 2009, A\&A, 507, 1393

Bica E., Ortolani S., Barbuy B., 2016, PASA, 33, e028

Bisterzo S., Gallino R., Straniero O., Cristallo S., Käppeler F., 2011, MNRAS, 418, 284

Bisterzo S., Travaglio C., Gallino R., Wiescher M., Käppeler F., 2014, ApJ, 787, 10

Bisterzo S., Travaglio C., Wiescher M., Käppeler F., Gallino R., 2017, ApJ, 835, 97

Bressan A., Marigo P., Girardi L., Salasnich B., Dal Cero C., Rubele S., Nanni A., 2012, MNRAS, 427, 127

Campos F., Kepler S. O., Bonatto C., Ducati J. R., 2013, MNRAS, 433, 243

Cantiello M. et al., 2009, A\&A, 499, 279

Carpenter J. M., 2001, AJ, 121, 2851

Carretta E., 2006, AJ, 131, 1766

Carretta E., Cohen J. G., Gratton R. G., Behr B. B., 2001, AJ, 122, 1469

Carretta E., Gratton R. G., Bragaglia A., Bonifacio P., Pasquini L., 2004 , A\&A, 416, 925

Carretta E. et al., 2007, A\&A, 464, 967

Carretta E. et al., 2009a, A\&A, 505, 117

Carretta E., Bragaglia A., Gratton R., Lucatello S., 2009b, A\&A, 505, 139

Carretta E., Bragaglia A., Gratton R. G., Recio-Blanco A., Lucatello S., D’Orazi V., Cassisi S., 2010, A\&A, 516, A55

Carretta E., Gratton R. G., Bragaglia A., D’Orazi V., Lucatello S., 2013, A\&A, 550, A34

Castelli F., Gratton R. G., Kurucz R. L., 1997, A\&A, 318, 841

Cescutti G., Matteucci F., Lanfranchi G. A., McWilliam A., 2008, A\&A, 491, 401

Charbonnel C., 2016, in Moraux E., Lebreton Y., Charbonnel C., eds, EAS Publications Series Vol. 80, p 177 (arXiv:1611.08855)

Cohen J. G., Gratton R. G., Behr B. B., Carretta E., 1999, ApJ, 523, 739

Cordero M. J., Pilachowski C. A., Johnson C. I., McDonald I., Zijlstra A. A., Simmerer J., 2014, ApJ, 780, 94

Cordero M. J., Pilachowski C. A., Johnson C. I., Vesperini E., 2015, ApJ, 800,3

D’Ercole A., Vesperini E., D’Antona F., McMillan S. L. W., Recchi S., 2008, MNRAS, 391, 825

D’Orazi V., Gratton R., Lucatello S., Carretta E., Bragaglia A., Marino A. F., 2010, ApJ, 719, L213

Da Costa G. S., Armandroff T. E., 1995, AJ, 109, 2533

Da Costa G. S., Seitzer P., 1989, AJ, 97, 405

de Mink S. E., Pols O. R., Langer N., Izzard R. G., 2009, A\&A, 507, L1

Decressin T., Meynet G., Charbonnel C., Prantzos N., Ekström S., 2007, A\&A, 464, 1029

Decressin T., Baumgardt H., Charbonnel C., Kroupa P., 2010, A\&A, 516, A73

Dekker H., D’Odorico S., Kaufer A., Delabre B., Kotzlowski H., 2000, in Iye M., Moorwood A. F., eds, Proc. SPIE Conf. Ser. Vol. 4008, Optical and IR Telescope Instrumentation and Detectors. p 534 
Denisenkov P. A., Denisenkova S. N., 1990, Soviet Astron. Lett., 16, 275

Denissenkov P. A., Hartwick F. D. A., 2014, MNRAS, 437, L21

Dias B., Barbuy B., Saviane I., Held E. V., Da Costa G. S., Ortolani S., Gullieuszik M., Vásquez S., 2016, A\&A, 590, A9

Dinescu D. I., Girard T. M., van Altena W. F., 1999, AJ, 117, 1792

Donati J.-F., Semel M., Carter B. D., Rees D. E., Collier Cameron A., 1997, MNRAS, 291, 658

Drout M. R. et al., 2017, Science

Eichler D., Livio M., Piran T., Schramm D. N., 1989, Nature, 340, 126

Elsner R. F. et al., 2008, ApJ, 687, 1019

Feltzing S., Primas F., Johnson R. A., 2009, A\&A, 493, 913

Freiburghaus C., Rosswog S., Thielemann F.-K., 1999, ApJ, 525, L121

Frenk C. S., White S. D. M., 1980, MNRAS, 193, 295

Frischknecht U., Hirschi R., Thielemann F.-K., 2012, A\&A, 538, L2

Frischknecht U. et al., 2016, MNRAS, 456, 1803

Gratton R. G. et al., 2001, A\&A, 369, 87

Gratton R., Sneden C., Carretta E., 2004, ARA\&A, 42, 385

Gratton R. G., Lucatello S., Bragaglia A., Carretta E., Momany Y., Pancino E., Valenti E., 2006, A\&A, 455, 271

Gratton R. G., Carretta E., Bragaglia A., 2012, A\&A Rev., 20, 50

Gratton R. G. et al., 2015, A\&A, 573, A92

Harris W. E., 1996, AJ, 112, 1487

Hartwick F. D. A., 1968, ApJ, 154, 475

Hesser J. E., Bell R. A., 1980, ApJ, 238, L149

Ishigaki M. N., Aoki W., Chiba M., 2013, ApJ, 771, 67

James G., François P., Bonifacio P., Carretta E., Gratton R. G., Spite F., 2004, A\&A, 427, 825

Johnson C. I., Rich R. M., Kobayashi C., Kunder A., Koch A., 2014, AJ, 148,67

Johnson C. I., Caldwell N., Rich R. M., Pilachowski C. A., Hsyu T., 2016, AJ, 152, 21

Karakas A. I., Lattanzio J. C., 2014, PASA, 31, e030

Kobayashi C., Nomoto K., 2009, ApJ, 707, 1466

Kobayashi C., Umeda H., Nomoto K., Tominaga N., Ohkubo T., 2006, ApJ, 653,1145

Krause M. G. H., Charbonnel C., Bastian N., Diehl R., 2016, A\&A, 587, A53

Langer G. E., Hoffman R., Sneden C., 1993, PASP, 105, 301

Larsen S. S., Baumgardt H., Bastian N., Brodie J. P., Grundahl F., Strader J., 2015, ApJ, 804, 71

Lattimer J. M., Schramm D. N., 1974, ApJ, 192, L145

Lind K., Charbonnel C., Decressin T., Primas F., Grundahl F., Asplund M., 2011, A\&A, 527, A148

Lind K., Bergemann M., Asplund M., 2012, MNRAS, 427, 50

Marino A. F. et al., 2011, A\&A, 532, A8

Mashonkina L., Zhao G., 2006, A\&A, 456, 313

Massari D. et al., 2017, MNRAS, 468, 1249

McWilliam A., 1997, ARA\&A, 35, 503

McWilliam A., 2016, PASA, 33, e040

McWilliam A., Bernstein R. A., 2008, ApJ, 684, 326

McWilliam A., Rich R. M., 1994, ApJS, 91, 749

Meléndez J., Cohen J. G., 2009, ApJ, 699, 2017

Mészáros S. et al., 2015, AJ, 149, 153

Milone A. P. et al., 2017, MNRAS, 464, 3636

Monelli M. et al., 2013, MNRAS, 431, 2126

Mucciarelli A. et al., 2016, ApJ, 824, 73

Nissen P. E., Schuster W. J., 2010, A\&A, 511, L10

Nomoto K., Kobayashi C., Tominaga N., 2013, ARA\&A, 51, 457

Paust N. E. Q. et al., 2009, AJ, 137, 246

Peñaloza F., Pessev P., Vaśquez S., Borissova J., Kurtev R., Zoccali M., 2015, PASP, 127, 329

Pignatari M., Gallino R., Heil M., Wiescher M., Käppeler F., Herwig F., Bisterzo S., 2010, ApJ, 710, 1557

Pike C. D., 1976, MNRAS, 177, 257

Ramírez S. V., Cohen J. G., 2002, AJ, 123, 3277

Reddy B. E., Lambert D. L., Allende Prieto C., 2006, MNRAS, 367, 1329

Renzini A. et al., 2015, MNRAS, 454, 4197
Rieke G. H., Lebofsky M. J., 1985, ApJ, 288, 618

Rosswog S., Korobkin O., Arcones A., Thielemann F.-K., Piran T., 2014, MNRAS, 439, 744

Salaris M., Cassisi S., Weiss A., 2002, The Publications of the Astronomical Society of the Pacific, 114, Issue 794, 375

Sarajedini A. et al., 2007, AJ, 133, 1658

Sariya D. P., Yadav R. K. S., 2015, A\&A, 584, A59

Simmerer J., Sneden C., Cowan J. J., Collier J., Woolf V. M., Lawler J. E., 2004, ApJ, 617, 1091

Skrutskie M. F. et al., 2006, AJ, 131, 1163

Sneden C., 1973, ApJ, 184, 839

Sneden C., Cowan J. J., Gallino R., 2008, ARA\&A, 46, 241

Sobeck J. S., Ivans I. I., Simmerer J. A., Sneden C., Hoeflich P., Fulbright J. P., Kraft R. P., 2006, AJ, 131, 2949

Stetson P. B., 2000, PASP, 112, 925

Tanvir N. R., Levan A. J., Fruchter A. S., Hjorth J., Hounsell R. A., Wiersema K., Tunnicliffe R. L., 2013, Nature, 500, 547

Thielemann F.-K. et al., 2011, Progr Particle Nucl. Phys., 66, 346

Thygesen A. O. et al., 2014, A\&A, 572, A108

Torres G., 2010, AJ, 140, 1158

Van der Swaelmen M., Barbuy B., Hill V., Zoccali M., Minniti D., Ortolani S., Gómez A., 2016, A\&A, 586, A1

Ventura P., D’Antona F., Mazzitelli I., Gratton R., 2001, ApJ, 550, L65

Ventura P., Criscienzo M. D., D'Antona F., Vesperini E., Tailo M., Dell'Agli F., D'Ercole A., 2014, MNRAS, 437, 3274

Vesperini E., McMillan S. L. W., D’Antona F., D’Ercole A., 2013, MNRAS, 429, 1913

Woosley S. E., Weaver T. A., 1995, ApJS, 101, 181

Woosley S. E., Heger A., Weaver T. A., 2002, Reviews of Modern Physics, 74,1015

Wylie E. C., Cottrell P. L., Sneden C. A., Lattanzio J. C., 2006, ApJ, 649, 248

Yong D. et al., 2014, MNRAS, 439, 2638

Zacharias N., Monet D. G., Levine S. E., Urban S. E., Gaume R., Wycoff G. L., 2004, BAAS, 36, 1418

Zoccali M., Pancino E., Catelan M., Hempel M., Rejkuba M., Carrera R., 2009, ApJ, 697, L22

\section{SUPPORTING INFORMATION}

Supplementary data are available at MNRAS online.

Table 2. Atomic line list: wavelength, ionization state (integer part: atomic number; decimal part:.0 for neutral and.1 for single ionized species), atomic parameters $\chi$ and $\log g f$ and EWs for each star.

Please note: Oxford University Press is not responsible for the content or functionality of any supporting materials supplied by the authors. Any queries (other than missing material) should be directed to the corresponding author for the article.

\section{APPENDIX: TABLES AND FIGURES}

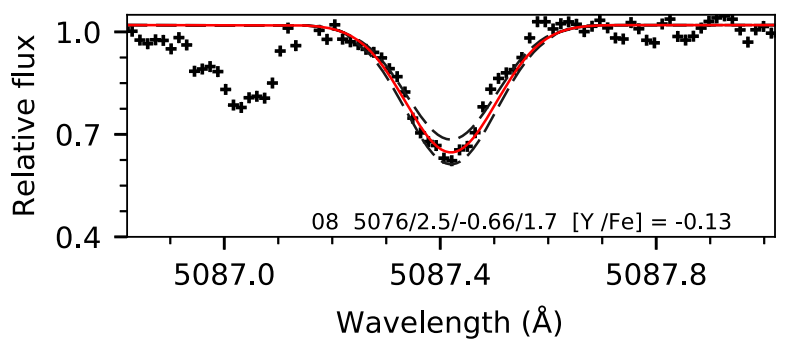

Figure A1. Example of spectral synthesis of the $5087 \AA$ line of Y. The symbols are as given in Fig. 5, except that only 1- $\sigma$ deviations are shown. 
Table A1. Uncertainties, in dex, for the elements measured by the EWs method.

\begin{tabular}{lcccccccc}
\hline Element & 01 & 03 & 05 & 06 & 07 & 08 & 11 & 18 \\
\hline Fe I & 0.19 & 0.19 & 0.21 & 0.19 & 0.20 & 0.19 & 0.19 & 0.20 \\
Fe II & 0.19 & 0.28 & 0.22 & 0.21 & 0.19 & 0.20 & 0.28 & 0.29 \\
O I & 0.14 & 0.14 & 0.18 & 0.16 & 0.16 & 0.14 & 0.14 & 0.16 \\
Na I & 0.15 & 0.13 & 0.13 & 0.13 & 0.13 & 0.15 & 0.18 & 0.19 \\
Mg I & 0.15 & 0.07 & 0.17 & 0.13 & 0.09 & 0.11 & 0.24 & 0.24 \\
Al I & 0.15 & 0.11 & 0.12 & 0.11 & 0.13 & 0.09 & 0.22 & 0.12 \\
Si I & 0.14 & 0.15 & 0.10 & 0.07 & 0.11 & 0.08 & 0.13 & 0.13 \\
Ca I & 0.18 & 0.20 & 0.18 & 0.19 & 0.19 & 0.16 & 0.21 & 0.20 \\
Sc II & 0.16 & 0.15 & 0.18 & 0.15 & 0.15 & 0.19 & 0.18 & 0.19 \\
Ti I & 0.24 & 0.24 & 0.24 & 0.22 & 0.22 & 0.22 & 0.28 & 0.26 \\
Ti II & 0.16 & 0.15 & 0.19 & 0.19 & 0.14 & 0.19 & 0.19 & 0.21 \\
V I & 0.27 & 0.28 & 0.25 & 0.23 & 0.29 & 0.27 & 0.33 & 0.35 \\
Cr I & 0.19 & $\ldots$ & 0.19 & 0.26 & $\ldots$ & 0.18 & 0.28 & 0.19 \\
Co I & 0.15 & 0.17 & 0.16 & 0.15 & 0.15 & 0.17 & 0.14 & 0.15 \\
Ni I & 0.20 & 0.17 & 0.19 & 0.19 & 0.19 & 0.17 & 0.19 & 0.21 \\
\hline
\end{tabular}

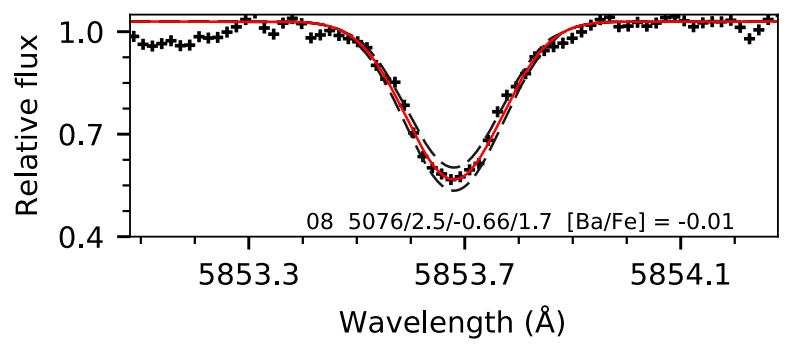

Figure A2. Example of spectral synthesis of the $5853 \AA$ line of Ba. The symbols are as given in from Fig. A1.

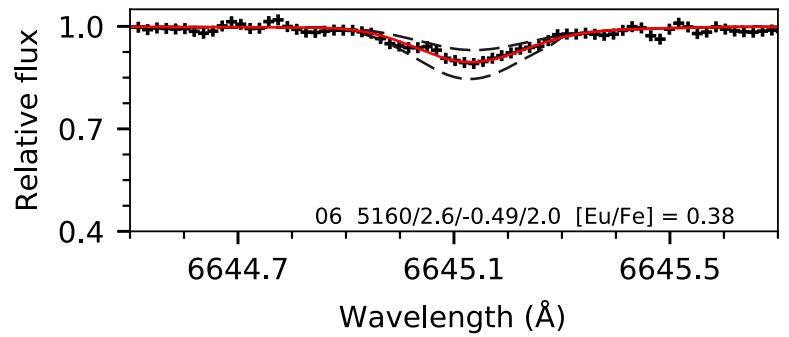

Figure A3. Example of spectral synthesis of the $6645 \AA$ line of Eu. The symbols are as given in Fig. A1.

This paper has been typeset from a $\mathrm{T}_{\mathrm{E}} \mathrm{X} / \mathrm{L} \mathrm{T}_{\mathrm{E}} \mathrm{X}$ file prepared by the author. 